\title{
SEPARATION PRINCIPLE IN THE FRACTIONAL GAUSSIAN LINEAR-QUADRATIC REGULATOR PROBLEM WITH PARTIAL OBSERVATION
}

\author{
Marina L. Kleptsyna ${ }^{1}$, Alain Le Breton ${ }^{2}$ and Michel Viot ${ }^{2}$
}

\begin{abstract}
In this paper we solve the basic fractional analogue of the classical linear-quadratic Gaussian regulator problem in continuous-time with partial observation. For a controlled linear system where both the state and observation processes are driven by fractional Brownian motions, we describe explicitly the optimal control policy which minimizes a quadratic performance criterion. Actually, we show that a separation principle holds, i.e., the optimal control separates into two stages based on optimal filtering of the unobservable state and optimal control of the filtered state. Both finite and infinite time horizon problems are investigated.
\end{abstract}

Mathematics Subject Classification. 93E11, 93E20. 60G15, 60G44.

Received July 21, 2006. Revised January 8, 2007.

\section{INTRODUCTION}

Several contributions in the literature have been already devoted to the extension of the classical theory of continuous-time stochastic systems driven by Brownian motions to analogues in which the driving processes are fractional Brownian motions (fBm's for short). The tractability of the basic problems in prediction, parameter estimation, filtering and control is now rather well understood (see, e.g., $[1,6-10,15,16]$, and references therein). Nevertheless, as far as we know, it is not yet demonstrated that optimal control problems can also be handled for fractional stochastic systems which are only partially observable. So, our aim here is to illustrate the actual solvability of such control problems by exhibiting an explicit solution for the case of the simplest linear-quadratic model.

We deal with the fractional analogue of the so-called linear-quadratic Gaussian regulator problem in one dimension. The real-valued processes $X=\left(X_{t}, t \geq 0\right)$ and $Y=\left(Y_{t}, t \geq 0\right)$, representing the state dynamic and the available observation record, respectively, are governed by the following linear system of stochastic

Keywords and phrases. Fractional Brownian motion, linear system, optimal control, optimal filtering, quadratic payoff, separation principle.

1 Laboratoire de Statistique et Processus, Université du Maine, av. Olivier Messiaen, 72085 Le Mans cedex 9, France; Marina.Kleptsyna@univ-lemans.fr

${ }^{2}$ Laboratoire de Modélisation et Calcul, Université J. Fourier, BP 53, 38041 Grenoble cedex 9, France; Alain.Le-Breton@imag.fr

(C) EDP Sciences, SMAI 2008 
differential equations, which shall be as usual interpreted as integral equations:

$$
\left\{\begin{aligned}
\mathrm{d} X_{t} & =a(t) X_{t} \mathrm{~d} t+b(t) u_{t} \mathrm{~d} t+\sigma(t) \mathrm{d} V_{t}^{H}, t \geq 0, X_{0}=x, \\
\mathrm{~d} Y_{t} & =A(t) X_{t} \mathrm{~d} t+B(t) \mathrm{d} W_{t}^{H}, t \geq 0, Y_{0}=0 .
\end{aligned}\right.
$$

Here $V=\left(V_{t}^{H}, t \geq 0\right)$ and $W=\left(W_{t}^{H}, t \geq 0\right)$ are independent normalized fBm's with Hurst parameter $H$ in $[1 / 2,1)$ and $x$ a fixed initial condition. The coefficients $a, b, \sigma, A$ and $B$ are assumed to be bounded and smooth enough (deterministic) functions of $\mathbb{R}^{+}$into $\mathbb{R}$, with $B$ nonvanishing and $B^{-1}$ bounded. We suppose that at each time $t \geq 0$ one may choose the input $u_{t}$ in view of the past observations $\left\{Y_{s}, 0 \leq s \leq t\right\}$ in order to drive the corresponding state, $X_{t}=X_{t}^{u}$ say, which hence also acts upon the observation, $Y_{t}=Y_{t}^{u}$ say. Then, given a cost function which evaluates the performance of the control actions, the classical problem of controlling the system dynamics on some time interval so as to minimize this cost occurs.

Here, in the finite time horizon case, given some fixed $T>0$, we consider the quadratic payoff $J_{T}$ defined for a control policy $u=\left(u_{t}, t \in[0, T]\right)$ by

$$
J_{T}(u)=\mathbb{E}\left\{q_{T} X_{T}^{2}+\int_{0}^{T}\left[q(t) X_{t}^{2}+r(t) u_{t}^{2}\right] \mathrm{d} t\right\},
$$

where $q_{T}$ is a positive constant and $q=(q(t), t \in[0, T])$ and $r=(r(t), t \in[0, T])$ are fixed (deterministic) positive continuous functions. It is well-known that when $H=1 / 2$ and hence the noises in (1) are Brownian motions, then (see, e.g., $[3,13,17]$ ), the solution $\bar{u}$ to the corresponding problem, called the optimal control, is provided for all $t \in[0, T]$ by

$$
\bar{u}_{t}=-\frac{b(t)}{r(t)} \rho(t) \pi_{t}(\bar{X}) ; \quad \bar{X}_{t}=X_{t}^{\bar{u}} ; \quad \bar{Y}_{t}=Y_{t}^{\bar{u}}
$$

where $\pi_{t}(\bar{X})$ is the conditional mean of $\bar{X}_{t}$ given $\left\{\bar{Y}_{s}, 0 \leq s \leq t\right\}$ and $\rho=(\rho(t), t \in[0, T])$ is the unique nonnegative solution of the backward Riccati differential equation

$$
\dot{\rho}(t)=-2 a(t) \rho(t)-q(t)+\frac{b^{2}(t)}{r(t)} \rho^{2}(t) ; \quad \rho(T)=q_{T} .
$$

In (3), the optimal filter $\pi_{t}(\bar{X})$ is generated by the following so-called Kalman-Bucy system on $[0, T]$ :

$$
\left\{\begin{aligned}
\mathrm{d} \pi_{t}(\bar{X}) & =\left[a(t) \pi_{t}(\bar{X})+b(t) \bar{u}_{t}\right] \mathrm{d} t+\frac{A(t)}{B^{2}(t)} \gamma(t)\left[\mathrm{d} \bar{Y}_{t}-A(t) \pi_{t}(\bar{X}) \mathrm{d} t\right], \pi_{0}(\bar{X})=x \\
\dot{\gamma}(t) & =2 a(t) \gamma(t)+\sigma^{2}(t)-\frac{A^{2}(t)}{B^{2}(t)} \gamma^{2}(t), \gamma(0)=0
\end{aligned}\right.
$$

where the solution $\gamma(t)$ of the last forward Riccati equation is nothing but the variance $\mathbb{E}\left(\bar{X}_{t}-\pi_{t}(\bar{X})\right)^{2}$ of the filtering error. Moreover, the minimal cost $J_{T}(\bar{u})$ is given by

$$
J_{T}(\bar{u})=\rho(0) x^{2}+\int_{0}^{T} \frac{A^{2}(t)}{B^{2}(t)} \rho(t) \gamma^{2}(t) \mathrm{d} t+q_{T} \gamma(T)+\int_{0}^{T} q(t) \gamma(t) \mathrm{d} t .
$$

This result is known as the separation or certainty-equivalence principle. It means that, optimally, the processing device which takes the observation record $\left\{\bar{Y}_{s}, 0 \leq s \leq t\right\}$ and converts it into a control value $\bar{u}_{t}$ separates into two stages: computation of the statistic $\pi_{t}(\bar{X})$ and computation of the control value $\bar{u}_{t}$ as a function of this statistic. The main feature is that these operations are independent in the sense that the Kalman-Bucy filter does not depend in any way on the coefficients $q_{T}, q, r$ defining the control problem, whereas the control function 
does not depend on the noise parameters $\sigma, B$, i.e., the controller behaves as if $\pi_{t}(\bar{X})$ were the actual state $\bar{X}_{t}$, which explains the term "certainty-equivalence". Our first goal here is to show that actually when the system (1) is driven by fBm's with some $H \in(1 / 2,1)$ instead of Brownian motions, an explicit solution to the optimal control problem under the performance criterion (2) is still available in terms of a kind of separation principle. One of the crucial points is that, as in the case $H=1 / 2$, again for $H \in(1 / 2,1)$ the original problem can be reduced to the optimal control problem with complete observation of the filtered state $\pi_{t}(X)$. This can be rather easily noticed thanks to the obvious orthogonality relation $\mathbb{E}\left(X_{t}-\pi_{t}(X)\right) u_{t}=0$ for any suitable variable $u_{t}$ which is measurable with respect to $\left\{Y_{s}, 0 \leq s \leq t\right\}$. Then, to solve the optimal control problem concerning $\pi_{t}(X)$, we adapt the approach led in [10] to address the case of complete observation in a linear-quadratic regulator problem with a fractional Brownian perturbation.

Actually, we shall deal also with the infinite time horizon problem. In this case, we assume that the coefficients $a, b, \sigma, A$ and $B$ in (1) are fixed constants and we choose an average quadratic payoff per unit time $J_{\infty}$ which is defined for a control policy $u=\left(u_{t}, t \geq 0\right)$ by

$$
J_{\infty}(u)=\limsup _{T \rightarrow+\infty} \frac{1}{T} \int_{0}^{T}\left[q X_{t}^{2}+r u_{t}^{2}\right] \mathrm{d} t,
$$

where $q$ and $r$ are positive constants. Here, it is well-known that when $H=1 / 2$, then (see, e.g., [3]) to get an optimal control $\bar{u}$, one has just, in the solution of the finite time horizon problem, to substitute for the function $\bar{\rho}$, given by (4), the nonnegative solution $\bar{\rho}$ of the algebraic Riccati equation $-2 a \bar{\rho}-q+\frac{b^{2}}{r} \bar{\rho}^{2}=0$, i.e.,

$$
\bar{\rho}=\frac{r}{b^{2}}\left[a+\delta_{c}\right] ; \quad \delta_{c}=\sqrt{a^{2}+\frac{b^{2}}{r} q} .
$$

In other words, $\bar{u}$ is provided for all $t \geq 0$ by

$$
\bar{u}_{t}=-\frac{b}{r} \bar{\rho} \pi_{t}(\bar{X}) ; \quad \bar{X}_{t}=X_{t}^{\bar{u}} ; \quad \bar{Y}_{t}=Y_{t}^{\bar{u}}
$$

where the optimal filter $\pi_{t}(\bar{X})$ is still generated by (5). So, again a separation principle holds. Moreover the optimal cost $J_{\infty}(\bar{u})$ is given by

$$
J_{\infty}(\bar{u})=\frac{A^{2}}{B^{2}} \bar{\rho} \bar{\gamma}^{2}+q \bar{\gamma} \quad \text { a.s. },
$$

where $\bar{\gamma}$ is the nonnegative solution of the algebraic Riccati equation $2 a \gamma+\sigma^{2}-\frac{A^{2}}{B^{2}} \gamma^{2}=0$, i.e.,

$$
\bar{\gamma}=\frac{B^{2}}{A^{2}}\left[a+\delta_{f}\right] ; \quad \delta_{f}=\sqrt{a^{2}+\frac{A^{2}}{B^{2}} \sigma^{2}} .
$$

We shall also extend these results to the case $H \in(1 / 2,1)$. Again, the main idea of the approach is that the original problem can be reduced to the optimal control problem with complete observation of the filtered state $\pi_{t}(X)$. But, contrarily to the finite time horizon case, here one of the difficult points is to obtain an "orthogonality" condition in the sense that the limit as $T$ tends to infinity of a time average $\frac{1}{T} \int_{0}^{T}\left(X_{t}-\pi_{t}(X)\right) u_{t} \mathrm{~d} t$ is equal to zero almost surely for any suitable process $u_{t}$. The verification of that condition and the analysis of several other crucial ergodic type properties require the precise study of the asymptotic behavior of various processes which have complicate structures. Then, to solve the optimal control problem concerning $\pi_{t}(X)$, we adapt the approach led in [12] to address the case of complete observation in a linear-quadratic regulator problem with a fractional Brownian perturbation.

The paper is organized as follows. At first in Section 1, we fix some notations and preliminaries. In particular, we associate to the original problems auxiliary filtering and control problems concerning Volterra type integral 
dynamics driven by appropriate Gaussian martingales corresponding to the fractional noises. Then, in Sections 3 and 4, the finite time and the infinite time horizon control problems are solved, respectively. Section 5 is devoted to a complementary analysis of the second case for which another optimal control defined in terms of a simpler and more explicit linear feedback is described. Finally, an Appendix in Section 5 is dedicated to auxiliary developments: we derive some technical results and we investigate ergodic type properties of some processes.

\section{Preliminaries}

\subsection{Terminology and notations}

In what follows all random variables and processes are defined on a given stochastic basis $\left(\Omega, \mathcal{F},\left(\mathcal{F}_{t}\right), \mathbb{P}\right)$. Moreover the natural filtration of a process is understood as the $\mathbb{P}$-completion of the filtration generated by this process.

Here, for some $H \in[1 / 2,1), B^{H}=\left(B_{t}^{H}, t \geq 0\right)$ is a normalized fractional Brownian motion with Hurst parameter $H$ means that $B^{H}$ is a Gaussian process with continuous paths such that $B_{0}^{H}=0, \mathbb{E} B_{t}^{H}=0$ and

$$
\mathbb{E} B_{s}^{H} B_{t}^{H}=\frac{1}{2}\left[s^{2 H}+t^{2 H}-|s-t|^{2 H}\right], s, t \geq 0 .
$$

Of course the $\mathrm{fBm}$ reduces to the standard Brownian motion when $H=1 / 2$. For $H \neq 1 / 2$, the fBm is outside the world of semimartingales but a theory of stochastic integration w.r. to fBm has been developed (see, e.g., [4] or [5]). Actually the case of deterministic integrands, which is sufficient for the purpose of the present paper, is easy to handle (see, e.g., [15]).

- Fundamental martingale associated to $B^{H}$. There are simple integral transformations which change the fBm to martingales (see $[9,15,16])$. In particular, defining for $0<s<t$

$$
\begin{gathered}
k_{H}(t, s)=\kappa_{H}^{-1} s^{\frac{1}{2}-H}(t-s)^{\frac{1}{2}-H} ; \kappa_{H}=2 H \Gamma\left(\frac{3}{2}-H\right) \Gamma\left(H+\frac{1}{2}\right), \\
w_{t}^{H}=\lambda_{H}^{-1} t^{2-2 H} ; \quad \lambda_{H}=\frac{2 H \Gamma(3-2 H) \Gamma\left(H+\frac{1}{2}\right)}{\Gamma\left(\frac{3}{2}-H\right)} \\
B_{t}^{*}=\int_{0}^{t} k_{H}(t, s) \mathrm{d} B_{s}^{H}
\end{gathered}
$$

then the process $B^{*}$ is a Gaussian martingale, called in [15] the fundamental martingale, whose variance function $\left\langle B^{*}\right\rangle$ is nothing but the function $w^{H}$. Actually, the natural filtration of $B^{*}$ coincides with the natural filtration $\left(\mathcal{B}_{t}^{H}\right)$ of $B^{H}$. In particular, we have the direct consequence of the results of [9] that, given a suitably regular deterministic function $g=(g(t), t \geq 0)$, the following representation holds:

$$
\int_{0}^{t} g(s) \mathrm{d} B_{s}^{H}=\int_{0}^{t} K_{H}^{g}(t, s) \mathrm{d} B_{s}^{*}
$$

where for $H \in(1 / 2,1)$ the function $K_{H}^{g}$ is given by

$$
K_{H}^{g}(t, s)=H(2 H-1) \int_{s}^{t} g(r) r^{H-\frac{1}{2}}(r-s)^{H-\frac{3}{2}} \mathrm{~d} r, 0 \leq s \leq t,
$$

and for $H=1 / 2$ the convention $K_{1 / 2}^{g}(t,.) \equiv g$ for all $t$ is used.

- Admissible controls. Let $\mathcal{U}$ be the class of $\left(\mathcal{F}_{t}\right)$-adapted processes $u=\left(u_{t}\right)$ such that the stochastic differential system (1) has a unique strong solution $\left(X^{u}, Y^{u}\right)$ which satisfies $J(u)<+\infty$, where, according to the setting, the cost $J(u)$ is evaluated by (2) or (7), with $X=X^{u}$. Actually, as mentioned in Section 1 , for control purpose 
we are interested in policies such that, for each $t, u_{t}$ depends only on the past observations $\left\{Y_{s}, 0 \leq s \leq t\right\}$. So, we introduce the class of admissible controls as the class $\mathcal{U}_{a d}$ of those $u$ 's in $\mathcal{U}$ which are $\left(\mathcal{Y}_{t}^{u}\right)$-adapted processes where $\left(\mathcal{Y}_{t}^{u}\right)$ is the natural filtration of the corresponding observation process $Y=Y^{u}$. For $u \in \mathcal{U}_{a d}$, the triple $\left(u, X^{u}, Y^{u}\right)$ is called an admissible triple and if $\bar{u} \in \mathcal{U}_{a d}$ is such that

$$
J(\bar{u})=\inf \left\{J(u), u \in \mathcal{U}_{a d}\right\},
$$

then it is called an optimal control and $(\bar{u}, \bar{X}, \bar{Y})$, where $\bar{X}=X^{\bar{u}}, \bar{Y}=Y^{\bar{u}}$, is called an optimal triple and the quantity $J(\bar{u})$ is called the optimal cost.

\subsection{Auxiliary filtering and control problems}

Of course, taking into account the results recalled in Section 1 for the case $H=1 / 2$, our guess is that again, in the fractional world, the optimal controller behaves as if the filtered state were the actual state. Actually, as a consequence of the orthogonality relation $\mathbb{E}\left(X_{t}-\pi_{t}(X)\right) u_{t}=0$ for $u$ 's belonging to $\mathcal{U}_{a d}$, one gets that $J_{T}(u)$ given by (2) can be decomposed as

$$
\begin{aligned}
J_{T}(u)= & \mathbb{E}\left\{q_{T} \pi_{T}^{2}(X)+\int_{0}^{T}\left[q(t) \pi_{t}^{2}(X)+r(t) u_{t}^{2}\right] \mathrm{d} t\right\} \\
& +q_{T} \mathbb{E}\left(X_{T}-\pi_{T}(X)\right)^{2}+\int_{0}^{T} q(t) \mathbb{E}\left(X_{t}-\pi_{t}(X)\right)^{2} \mathrm{~d} t .
\end{aligned}
$$

Hence, since in fact the variances $\mathbb{E}\left(X_{t}-\pi_{t}(X)\right)^{2}$ of the filtering errors do not depend on the specific control $u$, it appears that optimizing $J_{T}(u)$ is equivalent to minimizing the first expectation in the right hand side above, which expectation depends only on the filtered state $\pi_{t}(X)$. Now, it is clear that the solution of the filtering problem in model (1) and the solution of the control problem with complete observation in the corresponding model for $\pi_{t}(X)$ will be crucial in our analysis. So, we give some preliminary results about these two problems, adapting to the present context some of our previous works.

- Filtering problem. Actually, the filtering problem in model (1), without the additional term $b(t) u_{t}$ in the state dynamic, has been solved in [9]. But, in the present setting, since only $u$ 's belonging to $\mathcal{U}_{a d}$ are involved, it is rather immediate to extend the result in the following terms. With $k_{H}$ given by (13), we introduce the observation fundamental semimartingale $Z$ which is defined from $Y$ by:

$$
Z_{t}=\int_{0}^{t} k_{H}(t, s) B^{-1}(s) \mathrm{d} Y_{s} .
$$

It can be represented as

$$
Z_{t}=\int_{0}^{t} Q(s) \mathrm{d} w_{s}^{H}+W_{t}^{*}
$$

where $W^{*}$ is the Gaussian martingale associated to $W^{H}$ through (15) and

$$
Q(t)=\frac{\mathrm{d}}{\mathrm{d} w_{t}^{H}} \int_{0}^{t} k_{H}(t, s) \frac{A(s)}{B(s)} X_{s} \mathrm{~d} s,
$$

with a derivative understood in the sense of absolute continuity. The natural filtrations $\left(\mathcal{Z}_{t}\right)$ and $\left(\mathcal{Y}_{t}\right)$ of $Z$ and $Y$ coincide and moreover the innovation type process $\nu=\left(\nu_{t}, t \in[0, T]\right)$ can be defined as follows. Using, for any process $\xi=\left(\xi_{t} ; t \geq 0\right)$ such that $\mathbb{E}\left|\xi_{t}\right|<+\infty$, the notation $\pi_{t}(\xi)$ for the conditional expectation $\mathbb{E}\left(\xi_{t} / \mathcal{Y}_{t}\right)$ of $\xi_{t}$ given the $\sigma$-field $\mathcal{Y}_{t}$ (or equivalently $\mathcal{Z}_{t}$ ), the process $\nu$ is given by:

$$
\nu_{t}=Z_{t}-\int_{0}^{t} \pi_{s}(Q) \mathrm{d} w_{s}^{H}
$$


where $w^{H}, Z$ and $Q$ are given by (14), (18) and (19), respectively. Actually, $\nu$ does not depend on the admissible control $u$ and moreover it is a continuous Gaussian $\left(\mathcal{Y}_{t}\right)$-martingale, with the variance function $\langle\nu\rangle=w^{H}$, which allows a representation of the filter $\pi_{t}(X)$. We introduce the family of $2 \times 2$ matrix-valued deterministic functions $\Gamma_{f}(t,)=.\left(\Gamma_{f}(t, s), 0 \leq s \leq t\right)$ which satisfies the Riccati-Volterra type system

$$
\begin{aligned}
\Gamma_{f}(t, s) & =\int_{0}^{s}\left[\mathcal{A}_{f}(t, r) \Gamma_{f}^{\prime}(s, r)+\Gamma_{f}(t, r) \mathcal{A}_{f}^{\prime}(s, r)\right] \mathrm{d} r \\
& +\int_{0}^{s} \mathcal{C}(t, r) \mathcal{C}^{\prime}(s, r) \mathrm{d} w_{r}^{H}-\int_{0}^{s} \Gamma_{f}(t, r) \mathcal{E}_{2} \Gamma_{f}^{\prime}(s, r) \mathrm{d} w_{r}^{H}
\end{aligned}
$$

where the $2 \times 2$ matrices $\mathcal{A}_{f}(t, r), \mathcal{E}_{2}$ and vectors $\mathcal{C}(t, r)$ in $\mathbb{R}^{2}$ are given by

$$
\mathcal{A}_{f}(t, r)=a(r)\left(\begin{array}{cc}
1 & 0 \\
p(t, r) & 0
\end{array}\right) ; \quad \mathcal{E}_{2}=\left(\begin{array}{cc}
0 & 0 \\
0 & 1
\end{array}\right) ; \quad \mathcal{C}(t, r)=\left(\begin{array}{c}
K_{H}^{\sigma}(t, r) \\
q(t, r)
\end{array}\right)
$$

with

$$
p(t, r)=\frac{\mathrm{d}}{\mathrm{d} w_{t}^{H}} \int_{r}^{t} k_{H}(t, v) \frac{A(v)}{B(v)} \mathrm{d} v ; \quad q(t, r)=\frac{\mathrm{d}}{\mathrm{d} w_{t}^{H}} \int_{r}^{t} k_{H}(t, v) K_{H}^{\sigma}(v, r) \frac{A(v)}{B(v)} \mathrm{d} v .
$$

Then, the filter $\pi_{t}(X)$ is governed by

$$
\pi_{t}(X)=x+\int_{0}^{t} a(s) \pi_{s}(X) \mathrm{d} s+\int_{0}^{t} b(s) u_{s} \mathrm{~d} s+\int_{0}^{t} \Gamma_{f}^{12}(t, s) \mathrm{d} \nu_{s}
$$

where $\Gamma_{f}^{12}(t, s)$ is the $(1,2)$-entry of the matrix $\Gamma_{f}(t, s)$. Notice that the probabilistic interpretation of $\Gamma_{f}(t, s)$ is given in [9] (see also the beginning of section 6.2 in the Appendix below) and in particular, for $s=t$, the diagonal entries $\Gamma_{f}^{i i}(t, s)$ of $\Gamma_{f}(t, s)$ are nothing but the variances of the filtering errors for $X_{t}$ and $Q_{t}$, respectively, from $\left\{Y_{s}, 0 \leq s \leq t\right\}$, i.e.,

$$
\Gamma_{f}^{11}(t, t)=\mathbb{E}\left(X_{t}-\pi_{t}(X)\right)^{2} ; \quad \Gamma_{f}^{22}(t, t)=\mathbb{E}\left(Q_{t}-\pi_{t}(Q)\right)^{2} .
$$

Of course, since the definition (20) of the innovation $\nu_{t}$ involves the filter $\pi_{t}(Q)$, to generate the filter $\pi_{t}(X)$ from (23), one needs actually a complementary equation to form a closed system for the pair $\left(\pi_{t}(X), \pi_{t}(Q)\right)$. We shall provide such an equation below (see also [8] for a global system of filtering equations in the case of constant coefficients without control).

- Control problem. Now, from the discussion above, it becomes natural to analyze the control problem with complete observation of the state in a Volterra type dynamic inspired of (23). Precisely, we consider a state process $\Pi=\left(\Pi_{t}, t \geq 0\right)$ generated by

$$
\Pi_{t}=x+\int_{0}^{t} a(s) \Pi_{s} \mathrm{~d} s+\int_{0}^{t} b(s) u_{s} \mathrm{~d} s+\int_{0}^{t} \Gamma_{f}^{12}(t, s) \mathrm{d} M_{s},
$$

where $M$ is a Gaussian $\left(\mathcal{F}_{t}\right)$-martingale with the variance function $\langle M\rangle=w^{H}$. Here, in the control problem which is relevant regarding our original problem, the class of admissible controls $u$ is the whole class $\mathcal{U}$. Of course, according to the setting, the payoff $J(u)$ to minimize is evaluated by $(2)$ or $(7)$ with $\Pi=\Pi^{u}$ in place of $X$, respectively. We recognize that actually the just stated control problems are quite similar to those which have been solved in [10] and [12]. More precisely, to get here an optimal control $\bar{u}$, we have only to substitute $\Gamma_{f}^{12}(t, s)$ for $K_{H}^{c}(t, s)$ in the settings therein.

Therefore, in the finite horizon case, we introduce the family of $2 \times 2$ matrix-valued functions $\Gamma_{c}(., s)=$ $\left(\Gamma_{c}(t, s), t \in[s, T]\right)$ such that $\Gamma_{c}(., s)$ is the unique nonnegative symmetric solution of the backward Riccati 
differential equation in the variable $t$ on $[s, T]$

$$
\begin{aligned}
\dot{\Gamma}_{c}(t, s)= & -\mathcal{A}_{c}^{\prime}(t, s) \Gamma_{c}(t, s)-\Gamma_{c}(t, s) \mathcal{A}_{c}(t, s)-q(t) \mathcal{D}(t, s) \mathcal{D}^{\prime}(t, s) \\
& +\frac{b^{2}(t)}{r(t)} \Gamma_{c}(t, s) \mathcal{E}_{1} \Gamma_{c}(t, s) ; \quad \Gamma_{c}(T, s)=q_{T} \mathcal{D}(T, s) \mathcal{D}^{\prime}(T, s),
\end{aligned}
$$

where the $2 \times 2$ matrices $\mathcal{A}_{c}(t, s), \mathcal{E}_{1}$ and vectors $\mathcal{D}(t, s)$ in $\mathbb{R}^{2}$ are given by

$$
\mathcal{A}_{c}(t, s)=a(t)\left(\begin{array}{cc}
1 & \Gamma_{f}^{12}(t, s) \\
0 & 0
\end{array}\right) ; \quad \mathcal{E}_{1}=\left(\begin{array}{cc}
1 & 0 \\
0 & 0
\end{array}\right) ; \quad \mathcal{D}(t, s)=\left(\begin{array}{c}
1 \\
\Gamma_{f}^{12}(t, s)
\end{array}\right)
$$

Actually, the $(1,1)$-entry $\Gamma_{c}^{11}(t, s)$ of the matrix $\Gamma_{c}(t, s)$ does not depend on the variable $s$ and it is nothing but the solution $\rho(t)$ of the Riccati equation (4). Again, we shall denote by $\Gamma_{c}^{i j}(t, s)$ the $(i, j)$-entry of $\Gamma_{c}(t, s)$. Now, parallelling the developments in [10], we get an optimal control $\bar{u}$ in $\mathcal{U}$ such that the optimal pair $(\bar{u}, \bar{\Pi})$, where $\bar{\Pi}=\Pi^{\bar{u}}$, is governed on $[0, T]$ by the system

$$
\bar{u}_{t}=-\frac{b(t)}{r(t)}\left\{\rho(t) \bar{\Pi}_{t}+\int_{0}^{t}\left[\Gamma_{c}^{12}(t, s)-\rho(t) \Gamma_{f}^{12}(t, s)\right] \mathrm{d} M_{s}\right\} ; \quad \bar{\Pi}_{t}=\Pi_{t}^{\bar{u}},
$$

and moreover the optimal cost is

$$
J_{T}(\bar{v})=\rho(0) x^{2}+\int_{0}^{T} \Gamma_{c}^{22}(t, t) \mathrm{d} w_{t}^{H} .
$$

Similarly, in the infinite horizon time case, we shall be able to take benefit of the approach led in [12] in order to analyze the auxiliary control problem with complete observation associated with the original control problem.

\section{Finite time horizon CONTROL PROBlem}

At first we state our main result:

Theorem 2.1. Let $k_{H}(t, s), p(t, s)$ and $q(t, s)$ be the kernels defined in (13) and (22), respectively. Let also $\Gamma_{f}$, $\Gamma_{c}$ be the solutions of (21), (25), respectively, and $\bar{\rho}$ be the solution of (4). In the control problem

$$
\min _{u \in \mathcal{U}_{a d}} J_{T}(u) \text { subject to }(1)
$$

with $J_{T}$ defined by (2), an optimal control $\bar{u}$ in $\mathcal{U}_{a d}$ and the corresponding optimal triple $(\bar{u}, \bar{X}, \bar{Y})$ are governed on $[0, T]$ by the system

$$
\bar{u}_{t}=-\frac{b(t)}{r(t)}\left\{\rho(t) \pi_{t}(\bar{X})+\int_{0}^{t}\left[\Gamma_{c}^{12}(t, s)-\rho(t) \Gamma_{f}^{12}(t, s)\right] \mathrm{d} \nu_{s}\right\} ; \quad \bar{X}_{t}=X_{t}^{\bar{u}} ; \quad \bar{Y}_{t}=Y_{t}^{\bar{u}},
$$

where

$$
\nu_{t}=\int_{0}^{t} k_{H}(t, s) B^{-1}(s) \mathrm{d} \bar{Y}_{s}-\int_{0}^{t} \pi_{s}(\bar{Q}) \mathrm{d} w_{s}^{H},
$$

and the pair $\left(\pi_{t}(\bar{X}), \pi_{t}(\bar{Q})\right)$ is generated by

$$
\begin{gathered}
\pi_{t}(\bar{X})=x+\int_{0}^{t} a(s) \pi_{s}(\bar{X}) \mathrm{d} s \int_{0}^{t} b(s) \bar{u}_{s} \mathrm{~d} s+\int_{0}^{t} \Gamma_{f}^{12}(t, s) \mathrm{d} \nu_{s} \\
\pi_{t}(\bar{Q})=p(t, 0) x+\int_{0}^{t} a(s) p(t, s) \pi_{s}(\bar{X}) \mathrm{d} s+\int_{0}^{t} b(s) p(t, s) \bar{u}_{s} \mathrm{~d} s+\int_{0}^{t} \Gamma_{f}^{22}(t, s) \mathrm{d} \nu_{s} .
\end{gathered}
$$


Moreover the optimal cost is

$$
J_{T}(\bar{u})=\rho(0) x^{2}+\int_{0}^{T} \Gamma_{c}^{22}(t, t) \mathrm{d} w_{t}^{H}+q_{T} \Gamma_{f}^{11}(T, T)+\int_{0}^{T} q(t) \Gamma_{f}^{11}(t, t) \mathrm{d} t .
$$

Remark 2.2. (a) In the case $H=1 / 2$ where, for all $0 \leq s \leq t, k_{H}(t, s)=1, p(t, s)=q(t, s)=A(t) / B(t)$, it is easy to check that for all $0 \leq s \leq t \leq T$, the matrices $\Gamma_{f}(t, s)$ and $\Gamma_{c}(t, s)$ reduce to:

$$
\Gamma_{f}(t, s)=\gamma(s)\left(\begin{array}{cc}
1 & \frac{A(s)}{B(s)} \\
\frac{A(s)}{B(s)} & \frac{A^{2}(s)}{B^{2}(s)}
\end{array}\right) ; \quad \Gamma_{c}(t, s)=\rho(t)\left(\begin{array}{cc}
1 & \frac{A(s)}{B(s)} \gamma(s) \\
\frac{A(s)}{B(s)} \gamma(s) & \frac{A^{2}(s)}{B^{2}(s)} \gamma^{2}(s)
\end{array}\right)
$$

where $\rho$ and $\gamma$ are the solutions of the Riccati equations given in (4) and (5), respectively. Hence, it is readily seen that actually the statement in Theorem 2.1 reduces globally to the well-known result recalled in Section 1.

(b) Introducing

$$
\bar{v}_{t}=\int_{0}^{t}\left[\Gamma_{c}^{12}(t, s)-\rho(t) \Gamma_{f}^{12}(t, s)\right] \mathrm{d} \nu_{s},
$$

one can write the optimal control $\bar{u}_{t}$ as

$$
\bar{u}_{t}=-\frac{b(t)}{r(t)}\left\{\rho(t) \pi_{t}(\bar{X})+\bar{v}_{t}\right\}
$$

It is worth mentioning that actually the additional term $\bar{v}_{t}$ which appears in the case $H>1 / 2$ (and equals zero when $H=1 / 2$ ) can be interpreted in terms of the predictors at time $t$ of the noise component $V_{\tau}^{H}, t \leq \tau \leq T$ based on the observed optimal dynamics $\left(\bar{Y}_{s}, s \leq t\right)$ up to time $t$. Precisely, one can rewrite

$$
\bar{v}_{t}=\int_{t}^{T} \phi(\tau, t) \rho(\tau) \sigma(\tau) \frac{\partial}{\partial \tau} \mathbb{E}\left(V_{\tau}^{H} / \mathcal{F}_{t}^{\bar{u}}\right) \mathrm{d} \tau, t \leq \tau \leq T
$$

where

or, equivalently,

$$
\phi(\tau, t)=\exp \left\{\int_{t}^{\tau}\left[a(u)-\frac{b^{2}(u)}{r(u)} \rho(u)\right] \mathrm{d} u\right\},
$$

This will be made clear after the proof of Theorem 2.1 .

Proof of Theorem 2.1. Clearly, due to its definition through a closed system in terms of the only process $\bar{Y}$, the control policy $\bar{u}$ given by (28) belongs to $\mathcal{U}_{a d}$. Moreover, comparing (30) with (23), we see that $\pi_{t}(\bar{X})$ is nothing but the filter of $\bar{X}$ based on the observation of $\bar{Y}$. Actually, from the results in [9], it can be seen that $\pi_{t}(\bar{Q})$ given (31) is also the filter of the process $\bar{Q}$ which is the analogue for $\bar{X}$ of $Q$ defined from $X$ by (19). This explains why one may also substitute (29) for (20) in the representation of the innovation $\nu$ which does not depend on the admissible control. Let us consider the process $\left(p_{t}, 0 \leq t \leq T\right)$ defined by

$$
p_{t}=\rho(t) \pi_{t}(\bar{X})+\int_{0}^{t}\left[\Gamma_{c}^{12}(t, s)-\rho(t) \Gamma_{f}^{12}(t, s)\right] \mathrm{d} \nu_{s},
$$

which in particular allows the representation $\bar{u}_{t}=-(b(t) / r(t)) p_{t}$. Parallelling the proof in [10], it can be shown that it satisfies the following backward stochastic differential equation:

$$
\mathrm{d} p_{t}=-a(t) p_{t} \mathrm{~d} t-q(t) \pi_{t}(\bar{X}) \mathrm{d} t+\Gamma_{c}^{12}(t, t) \mathrm{d} \nu_{t}, t \in[0, T] ; p_{T}=q_{T} \pi_{T}(\bar{X}) .
$$


Now we show that $\bar{u}$ minimizes $J_{T}$ over $\mathcal{U}_{a d}$. Given an arbitrary $u \in \mathcal{U}_{a d}$, we evaluate the difference $J_{T}(u)-J_{T}(\bar{u})$ between the corresponding cost and the cost for the announced candidate $\bar{u}$ to optimality. Of course, we can write

$$
J_{T}(u)-J_{T}(\bar{u})=\mathbb{E}\left\{q_{T}\left[X_{T}^{2}-\bar{X}_{T}^{2}\right]+\int_{0}^{T}\left\{q(t)\left[X_{t}^{2}-\bar{X}_{t}^{2}\right]+r(t)\left[u_{t}^{2}-\bar{u}_{t}^{2}\right]\right\} \mathrm{d} t\right\} .
$$

Using the equality $y^{2}-\bar{y}^{2}=(y-\bar{y})^{2}+2 \bar{y}(y-\bar{y})$ and exploiting the property $\bar{u}=-(b / r) p$, it is readily seen that

where

$$
J_{T}(u)-J_{T}(\bar{u})=\mathbb{E}\left(\Delta_{1}\right)+2 \mathbb{E}\left(\Delta_{2}\right),
$$

$$
\begin{aligned}
& \Delta_{1}=q_{T}\left[X_{T}-\bar{X}_{T}\right]^{2}+\int_{0}^{T}\left\{q(t)\left[X_{t}-\bar{X}_{t}\right]^{2}+r(t)\left[u_{t}-\bar{u}_{t}\right]^{2}\right\} \mathrm{d} t, \\
& \Delta_{2}=q_{T} \bar{X}_{T}\left[X_{T}-\bar{X}_{T}\right]+\int_{0}^{T}\left\{q(t) \bar{X}_{t}\left[X_{t}-\bar{X}_{t}\right]-b(t) p_{t}\left[u_{t}-\bar{u}_{t}\right]\right\} \mathrm{d} t .
\end{aligned}
$$

Actually, the last integral can be written as

$$
\int_{0}^{T}\left\{\left(X_{t}-\bar{X}_{t}\right)\left[q(t) \bar{X}_{t}+a(t) p_{t}\right]-p_{t}\left[a(t)\left(X_{t}-\bar{X}_{t}\right)+b(t)\left(u_{t}-\bar{u}_{t}\right)\right]\right\} \mathrm{d} t
$$

and moreover, due to (1), (23) and (30), $X_{t}-\bar{X}_{t}=\pi_{t}(X)-\pi_{t}(\bar{X})$. Hence we can rewrite $\Delta_{2}$ in the form

$$
\begin{aligned}
\Delta_{2}= & q_{T}\left[\bar{X}_{T}-\pi_{T}(\bar{X})\right]\left[\pi_{T}(X)-\pi_{T}(\bar{X})\right]+\int_{0}^{T} q(t)\left[\bar{X}_{t}-\pi_{t}(\bar{X})\right]\left[\pi_{t}(X)-\pi_{t}(\bar{X})\right] \mathrm{d} t \\
& +q_{T} \pi_{T}(\bar{X})\left[\pi_{T}(X)-\pi_{T}(\bar{X})\right]+\int_{0}^{T}\left[\pi_{t}(X)-\pi_{t}(\bar{X})\right]\left[q(t) \pi_{t}(\bar{X})+a p_{t}\right] \mathrm{d} t \\
& -\int_{0}^{T} p_{t}\left[a(t)\left(\pi_{t}(X)-\pi_{t}(\bar{X})\right)+b(t)\left(u_{t}-\bar{u}_{t}\right)\right] \mathrm{d} t .
\end{aligned}
$$

Now, taking into account equation (36), we see that the difference of the last two integrals can be written as

$$
-\int_{0}^{T}\left(\pi_{t}(X)-\pi_{t}(\bar{X})\right) \mathrm{d} p_{t}-\int_{0}^{T} p_{t} d\left(\pi_{t}(X)-\pi_{t}(\bar{X})\right)+\int_{0}^{T}\left(\pi_{t}(X)-\pi_{t}(\bar{X})\right) \Gamma_{c}^{12}(t, t) \mathrm{d} \nu_{t} .
$$

Therefore, inserting this into the expression above of $\Delta_{2}$ and taking the expectation, since $\mathbb{E}\left[\bar{X}_{t}-\pi_{t}(\bar{X})\right]\left[\pi_{t}(X)-\right.$ $\left.\pi_{t}(\bar{X})\right]=0$ and the stochastic integral part gives also zero, we get that

$$
\mathbb{E}\left(\Delta_{2}\right)=\mathbb{E}\left\{q_{T} \pi_{T}(\bar{X})\left[\pi_{T}(X)-\pi_{T}(\bar{X})\right]-\int_{0}^{T}\left(\pi_{t}(X)-\pi_{t}(\bar{X})\right) \mathrm{d} p_{t}-\int_{0}^{T} p_{t} d\left(\pi_{t}(X)-\pi_{t}(\bar{X})\right)\right\} .
$$

Now, integrating by parts, since $p_{T}=q_{T} \pi_{T}(\bar{X})$ and $\pi_{0}(X)-\pi_{0}(\bar{X})=0$, it comes that $\mathbb{E}\left(\Delta_{2}\right)=0$ and finally $J_{T}(u)-J_{T}(\bar{u})=\mathbb{E}\left(\Delta_{1}\right) \geq 0$. This of course means that $\bar{u}$ minimizes $J_{T}$ over $\mathcal{U}_{a d}$.

Now we compute the optimal cost $J_{T}(\bar{u})$. Since

$$
\bar{X}_{t}^{2}=\pi_{t}^{2}(\bar{X})+\left[\bar{X}_{t}-\pi_{t}(\bar{X})\right]^{2}+2 \pi_{t}(\bar{X})\left[\bar{X}_{t}-\pi_{t}(\bar{X})\right],
$$

and $\mathbb{E}\left[\pi_{t}(\bar{X})\left(\bar{X}_{t}-\pi_{t}(\bar{X})\right)\right]=0$, we can write

$$
\begin{aligned}
J_{T}(\bar{u})= & \mathbb{E}\left\{q_{T} \pi_{T}^{2}(\bar{X})+\int_{0}^{T}\left[q(t) \pi_{t}^{2}(\bar{X})+r(t) \bar{u}_{t}^{2}\right] \mathrm{d} t\right\} \\
& +q_{T} \mathbb{E}\left[\bar{X}_{T}-\pi_{T}(\bar{X})\right]^{2}+\int_{0}^{T} q(t) \mathbb{E}\left[\bar{X}_{t}-\pi_{t}(\bar{X})\right]^{2} \mathrm{~d} t .
\end{aligned}
$$


Comparing (28), (30) with (24), (26), we recognize that the first quantity in the right-hand side above is nothing but the optimal cost $(27)$ in the auxiliary control problem discussed in Section 2. Therefore, since moreover $\mathbb{E}\left(\bar{X}_{t}-\pi_{t}(\bar{X})\right)^{2}=\Gamma_{f}^{11}(t, t)$, we see that the expression (32) holds for $J_{T}(\bar{u})$.

Justification of Remark 2.2.b Paralleling the discussion in [10], it follows that

$$
\bar{v}_{t}=\int_{t}^{T} \phi(\tau, t) \rho(\tau) \frac{\partial}{\partial \tau} \mathbb{E}\left(V_{\tau} / \mathcal{Y}_{t}^{\bar{u}}\right) \mathrm{d} \tau
$$

where $\phi(\tau, t)$ is given by $(34)$ and

$$
V_{\tau}=\int_{0}^{\tau} \Gamma_{f}^{12}(\tau, r) \mathrm{d} \nu_{r}
$$

But for any $\tau \geq t$ we have

$$
\mathbb{E}\left(V_{\tau} / \mathcal{Y}_{t}^{\bar{u}}\right)=\int_{0}^{t} \Gamma_{f}^{12}(\tau, r) \mathrm{d} \nu_{r}
$$

and hence also

$$
\frac{\partial}{\partial \tau} \mathbb{E}\left(V_{\tau} / \mathcal{Y}_{t}^{\bar{u}}\right)=\int_{0}^{t} \frac{\partial}{\partial \tau} \Gamma_{f}^{12}(\tau, r) \mathrm{d} \nu_{r}
$$

Therefore, the equality (33) will be valid if we prove

$$
\int_{0}^{t} \frac{\partial}{\partial \tau} \Gamma_{f}^{12}(\tau, r) \mathrm{d} \nu_{r}=\sigma(\tau) \frac{\partial}{\partial \tau} \mathbb{E}\left(V_{\tau}^{H} / \mathcal{Y}_{t}^{\bar{u}}\right) ; \quad \tau>t
$$

But for $\tau>t$ the following representation holds:

$$
\mathbb{E}\left(V_{\tau}^{H} / \mathcal{Y}_{t}^{\bar{u}}\right)=\int_{0}^{t}\left[\frac{\partial}{\partial\langle\nu\rangle_{r}} \mathbb{E} V_{\tau}^{H} \nu_{r}\right] \mathrm{d} \nu_{r}
$$

and so we just need to show that

$$
\frac{\partial}{\partial \tau} \Gamma_{f}^{12}(\tau, r)=\sigma(\tau) \frac{\partial}{\partial \tau} \frac{\partial}{\partial\langle\nu\rangle_{r}} \mathbb{E} V_{\tau}^{H} \nu_{r}
$$

To prove this equality, for $r<t<\tau$, we introduce the quantity

$$
G(\tau, r)=\frac{\partial}{\partial\langle\nu\rangle_{r}} \mathbb{E} X_{\tau}^{0} \nu_{r}
$$

where $X^{0}$ stands for $X^{u}$ with $u \equiv 0$. Since $\mathbb{E} \pi_{\tau}\left(X^{0}\right) \nu_{r}=\mathbb{E} X_{\tau}^{0} \nu_{r}$, from equations (23) and (1) (with $\left.u \equiv 0\right)$, we can derive the following two equations for $G(\tau, r)$ :

$$
\begin{gathered}
\frac{\partial}{\partial \tau} G(\tau, r)=a(\tau) G(\tau, r)+\frac{\partial}{\partial \tau} \Gamma_{f}^{12}(\tau, r), \\
\frac{\partial}{\partial \tau} G(\tau, r)=a(\tau) G(\tau, r)+\sigma(\tau) \frac{\partial}{\partial \tau} \frac{\partial}{\partial\left\langle\nu_{r}\right\rangle} \mathbb{E} V_{\tau}^{H} \nu_{r} .
\end{gathered}
$$

This gives that (37) holds and hence also (33). 


\section{INFINITE TIME HORIZON CONTROL PROBLEM - FiRST SOLUTION}

Here we assume that the coefficients $a, b, \sigma, A$ and $B$ in (1) are fixed constants and that $q$ and $r$ are positive constants. Then, in equation $(21)$ for $\Gamma_{f}(t, s)=\left(\left(\Gamma_{f}^{i j}(t, s)\right)\right)$, the coefficients $\mathcal{A}_{f}$ and $\mathcal{C}$ take the particular form

$$
\mathcal{A}_{f}(t, r)=a\left(\begin{array}{cc}
1 & 0 \\
\frac{A}{B} p^{*}(t, r) & 0
\end{array}\right) ; \quad \mathcal{C}(t, r)=\sigma\left(\begin{array}{c}
K_{H}(t, r) \\
\frac{A}{B} q^{*}(t, r)
\end{array}\right)
$$

with

$$
p^{*}(t, r)=\frac{\mathrm{d}}{\mathrm{d} w_{t}^{H}} \int_{r}^{t} k_{H}(t, v) \mathrm{d} v ; \quad q^{*}(t, r)=\frac{\mathrm{d}}{\mathrm{d} w_{t}^{H}} \int_{r}^{t} k_{H}(t, v) K_{H}(v, r) \mathrm{d} v,
$$

where $k_{H}$ is given by (13) and $K_{H}$ denotes $K_{H}^{g}$ defined by (17) when $g \equiv 1$, i.e.,

$$
K_{H}(t, s)=H(2 H-1) \int_{s}^{t} r^{H-\frac{1}{2}}(r-s)^{H-\frac{3}{2}} \mathrm{~d} r, 0 \leq s \leq t .
$$

Taking benefit of the approach led in [12] for the infinite time horizon control problem with complete observation, it is natural to introduce the following family of auxiliary functions $\left(\gamma_{c}^{12}(., s), s \geq 0\right)$. For any fixed $s \geq 0$, we define the function $\gamma_{c}^{12}(., s)=\left(\gamma_{c}^{12}(t, s), t \geq s\right)$ by

$$
\gamma_{c}^{12}(t, s)=\delta_{c} \mathrm{e}^{\delta_{c} t} \int_{t}^{+\infty} \mathrm{e}^{-\delta_{c} \tau} \Gamma_{f}^{12}(\tau, s) \mathrm{d} \tau
$$

where $\delta_{c}$ is given by (8). Now, we can state our main result:

Theorem 3.1. Let $k_{H}(t, s), p^{*}(t, s), q^{*}(t, s)$ and $\Gamma_{f}(t, s)$ be the kernels defined in (13), (39) and (21) with $\mathcal{A}_{f}$ and $\mathcal{C}$ given by (38). Let also the constants $\bar{\rho}, \bar{\gamma}$ be defined by (8), (11) and the function $\gamma_{c}^{12}$ be given by (40). In the control problem

$$
\min _{u \in \mathcal{U}_{a d}} J_{\infty}(u) \quad \text { subject to }(1)
$$

with $J_{\infty}$ defined by (7), an optimal control $\bar{u}$ in $\mathcal{U}_{\text {ad }}$ and the corresponding optimal triple $(\bar{u}, \bar{X}, \bar{Y})$ are governed by the system

$$
\bar{u}_{t}=-\frac{b}{r} \bar{\rho}\left\{\pi_{t}(\bar{X})+\int_{0}^{t}\left[\gamma_{c}^{12}(t, s)-\Gamma_{f}^{12}(t, s)\right] \mathrm{d} \nu_{s}\right\} ; \quad \bar{X}_{t}=X_{t}^{\bar{u}} ; \quad \bar{Y}_{t}=Y_{t}^{\bar{u}}
$$

where

$$
\nu_{t}=B^{-1} \int_{0}^{t} k_{H}(t, s) \mathrm{d} \bar{Y}_{s}-\int_{0}^{t} \pi_{s}(\bar{Q}) \mathrm{d} w_{s}^{H},
$$

and the pair $\left(\pi_{t}(\bar{X}), \pi_{t}(\bar{Q})\right)$ is generated by

$$
\begin{gathered}
\pi_{t}(\bar{X})=x+\int_{0}^{t} a \pi_{s}(\bar{X}) \mathrm{d} s+\int_{0}^{t} b \bar{u}_{s} \mathrm{~d} s+\int_{0}^{t} \Gamma_{f}^{12}(t, s) \mathrm{d} \nu_{s} \\
\pi_{t}(\bar{Q})=\frac{A}{B}\left\{p^{*}(t, 0) x+a \int_{0}^{t} p^{*}(t, s) \pi_{s}(\bar{X}) \mathrm{d} s+b \int_{0}^{t} p^{*}(t, s) \bar{u}_{s} \mathrm{~d} s\right\}+\int_{0}^{t} \Gamma_{f}^{22}(t, s) \mathrm{d} \nu_{s} .
\end{gathered}
$$

Moreover the optimal cost is

$$
J_{\infty}(\bar{u})=\zeta_{\infty}(H)+q \gamma_{\infty}(H)
$$


where

and

$$
\begin{aligned}
\zeta_{\infty}(H)= & \frac{A^{2}}{B^{2}} \bar{\rho}^{2} \frac{\Gamma(2 H+1) \sin \pi H}{\left(\delta_{f}-\delta_{c}\right)^{2}\left(\delta_{f}+\delta_{c}\right)}\left\{\left(\delta_{c}+a\right)\left[\frac{\delta_{f}-a}{\delta_{c}^{H-\frac{1}{2}}}-\frac{\delta_{c}-a}{\delta_{f}^{H-\frac{1}{2}}}\right]^{2}\right. \\
& \left.+\left(\delta_{f}-\delta_{c}\right)\left(\delta_{f}-a\right)\left[\frac{\delta_{f}-a}{\delta_{c}^{2 H-1}}-\frac{\delta_{c}-a}{\delta_{f}^{2 H-1}}\right]\right\} \\
& +\Gamma(2 H+1) \frac{q \sigma^{2}}{2}(1-\sin \pi H) \frac{\delta_{f}^{2}-a^{2}}{\delta_{f}^{2}-\delta_{c}^{2}}\left[\frac{1}{\delta_{c}^{2 H}}-\frac{1}{\delta_{f}^{2 H}}\right],
\end{aligned}
$$

$$
\gamma_{\infty}(H)=\frac{\sigma^{2} \Gamma(2 H+1)}{2 \delta_{f}^{2 H}}\left[1+\frac{\delta_{f}+a}{\delta_{f}-a} \sin \pi H\right],
$$

with $\delta_{c}, \delta_{f}$ given by (8), (11).

Remark 3.2. (a) From the proof below, it will appear that the term $\gamma_{\infty}(H)$, given by (47) which is involved in the representation (45) of the optimal cost $J_{\infty}(\bar{u})$, is nothing but the limit as $t$ tends to infinity of the variance $\mathbb{E}\left(\bar{X}_{t}-\pi_{t}(\bar{X})\right)^{2}$ of the filtering error.

Actually, in the statement above, for $\delta_{f}=\delta_{c}$, the quantity $\zeta_{\infty}(H)$ must be interpreted as the limit of the right hand side of (46) as $\delta_{c}$ tends to $\delta_{f}$. This limit is nothing but

$$
\begin{gathered}
\frac{A^{2}}{B^{2}} \bar{\rho} \bar{\gamma}^{2} \frac{\Gamma(2 H+1) \sin \pi H}{2 \delta_{f}^{2 H+2}}\left\{\left(\delta_{f}+a\right)\left[\delta_{f}+\left(H-\frac{1}{2}\right)\left(\delta_{f}-a\right)\right]^{2}+\delta_{f}\left(\delta_{f}-a\right)\left[\delta_{f}+(2 H-1)\left(\delta_{f}-a\right)\right]\right\} \\
+\Gamma(2 H+1) \frac{H q \sigma^{2}}{2 \delta_{f}^{2 H+2}}(1-\sin \pi H)\left(\delta_{f}^{2}-a^{2}\right) .
\end{gathered}
$$

(b) In the case $H=1 / 2$ where, for all $0 \leq s \leq t, k_{H}(t, s)=1, p^{*}(t, s)=q^{*}(t, s)=1$, it is easy to check that for all $0 \leq s \leq t \leq T$, the matrix $\Gamma_{f}(t, s)$ and the quantity $\gamma_{c}^{12}(t, s)$ reduce to:

$$
\Gamma_{f}(t, s)=\left(\begin{array}{cc}
1 & \frac{A}{B} \\
\frac{A}{B} & \frac{A^{2}}{B^{2}}
\end{array}\right) \gamma(s) ; \quad \gamma_{c}^{12}(t, s)=\frac{A}{B} \gamma(s),
$$

where $\gamma$ is the solution of the Riccati equation given in (5). Hence, it is readily seen that actually the statement in Theorem 3.1 reduces globally to the well-known result recalled in Section 1.

(c) Introducing

$$
\bar{v}_{t}=\int_{0}^{t}\left[\gamma_{c}^{12}(t, s)-\Gamma_{f}^{12}(t, s)\right] \mathrm{d} \nu_{s}
$$

one can write the optimal control $\bar{u}_{t}$ as

$$
\bar{u}_{t}=-\frac{b}{r} \bar{\rho}\left[\pi_{t}(\bar{X})+\bar{v}_{t}\right]
$$

It is worth mentioning that actually the additional term $\bar{v}_{t}$ which appears in the case $H>1 / 2$ (and equals zero when $H=1 / 2$ ) can be interpreted in terms of the predictors at time $t$ of the noise component $V_{\tau}^{H}, \tau \geq t$ based on the observed optimal dynamics $\left(\bar{Y}_{s}, s \leq t\right)$ up to time $t$. Precisely, one can rewrite

$$
\bar{v}_{t}=\sigma \int_{t}^{+\infty} \mathrm{e}^{-\delta_{c}(\tau-t)} \frac{\partial}{\partial \tau} \mathbb{E}\left(V_{\tau}^{H} / \mathcal{Y}_{t}^{\bar{u}}\right) \mathrm{d} \tau, \tau \geq t,
$$

or, equivalently,

$$
\bar{v}_{t}=\sigma \mathbb{E}\left(\int_{t}^{+\infty} \mathrm{e}^{-\delta_{c}(\tau-t)} \mathrm{d} V_{\tau}^{H} / \mathcal{Y}_{t}^{\bar{u}}\right) .
$$

This can be derived from the discussion in [12] by means of arguments similar to those which have been used above to prove (33). 
Proof of Theorem 3.1. Concerning the admissibility of $\bar{u}$ and the interpretation of the different terms involved in the system, one may repeat exactly the arguments at the beginning of the proof of Theorem 2.1. Let us consider the process $\left(p_{t}, t \geq 0\right)$ defined by

$$
p_{t}=\bar{\rho}\left\{\pi_{t}(\bar{X})+\int_{0}^{t}\left[\gamma_{c}^{12}(t, s)-\Gamma_{f}^{12}(t, s)\right] \mathrm{d} \nu_{s}\right\},
$$

which in particular allows the representation $\bar{u}_{t}=-(b / r) p_{t}$. Parallelling the proof in [12], it can be shown that it satisfies the following stochastic differential equation:

$$
\mathrm{d} p_{t}=-a p_{t} \mathrm{~d} t-q \pi_{t}(\bar{X}) \mathrm{d} t+\bar{\rho} \gamma_{c}^{12}(t, t) \mathrm{d} \nu_{t}, t \geq 0 ; p_{0}=\bar{\rho} x .
$$

Now we compute the cost $J_{\infty}(\bar{u})$ corresponding to the control $\bar{u}$. Given an arbitrary $u \in \mathcal{U}_{a d}$, we use the notation

$$
j_{T}(u)=\int_{0}^{T}\left[q X_{t}^{2}+r u_{t}^{2}\right] \mathrm{d} t
$$

where $X_{t}=X_{t}^{u}$. For $u=\bar{u}$, we can write

$$
\begin{aligned}
j_{T}(\bar{u})= & \int_{0}^{T}\left[q \pi_{t}^{2}(\bar{X})+r \bar{u}_{t}^{2}\right] \mathrm{d} t \\
& +q \int_{0}^{T}\left[\bar{X}_{t}-\pi_{t}(\bar{X})\right]^{2} \mathrm{~d} t+2 q \int_{0}^{T} \pi_{t}(\bar{X})\left[\bar{X}_{t}-\pi_{t}(\bar{X})\right] \mathrm{d} t .
\end{aligned}
$$

From (41), (43) and (51), we recognize that the pair $\left(\bar{u}_{t}, \pi_{t}(\bar{X})\right)$ is governed by a dynamic similar to that of the optimal pair obtained in [12] for an infinite horizon time problem under fractional Brownian perturbation and complete observation. Therein, the function $K_{H}(t, s)$ plays exactly the same role as $\Gamma_{f}^{12}(t, s)$ here. Then, we may parallel the developments (see section 6.3 in the Appendix below) to get the limit a.s.

$$
\lim _{T \rightarrow+\infty} \frac{1}{T} \int_{0}^{T}\left[q \pi_{t}^{2}(\bar{X})+r \bar{u}_{t}^{2}\right] \mathrm{d} t=\zeta_{\infty}(H),
$$

where $\zeta_{\infty}(H)$ is given by (46). Concerning the second term in $(52)$, of course again we have $\mathbb{E}\left(\bar{X}_{t}-\pi_{t}(\bar{X})\right)^{2}=$ $\Gamma_{f}^{11}(t, t)$. Moreover, the result obtained in [8] for the limiting behavior of this variance of the filtering error in an autonomous linear model driven by fractional Brownian noises tells that

$$
\lim _{t \rightarrow+\infty} \Gamma_{f}^{11}(t, t)=\gamma_{\infty}(H)
$$

where $\gamma_{\infty}(H)$ is given by (47). Actually, in the Appendix (cf. Sect. 6.3), we prove that the process $\bar{X}_{t}-\pi_{t}(\bar{X})$ possesses the following ergodic type property:

$$
\lim _{T \rightarrow+\infty} \frac{1}{T} \int_{0}^{T}\left[\bar{X}_{t}-\pi_{t}(\bar{X})\right]^{2} \mathrm{~d} t=\gamma_{\infty}(H), \quad \text { a.s. }
$$

Moreover we shall show that a.s.

$$
\lim _{T \rightarrow+\infty} \frac{1}{T} \int_{0}^{T} \pi_{t}(\bar{X})\left[\bar{X}_{t}-\pi_{t}(\bar{X})\right] \mathrm{d} t=0 .
$$

So, finally, the $\operatorname{limit} \lim _{T \rightarrow+\infty} \frac{1}{T} j_{T}(\bar{u})$ exists a.s. and inserting (53)-(55) into (52), we see that the expressio (45) holds for $J_{\infty}(\bar{u})$. 
Now we show that $\bar{u}$ minimizes $J_{\infty}$ over $\mathcal{U}_{a d}$. Proceeding along steps quite similar to those followed in the proof of Theorem 2.1, we can evaluate the difference $j_{T}(u)-j_{T}(\bar{u})$ as

$$
j_{T}(u)-j_{T}(\bar{u})=\Delta_{1}(T)+2 \Delta_{2}(T),
$$

where

$$
\Delta_{1}(T)=\int_{0}^{T}\left\{q\left[X_{t}-\bar{X}_{t}\right]^{2}+r\left[u_{t}-\bar{u}_{t}\right]^{2}\right\} \mathrm{d} t
$$

and

$$
\begin{aligned}
\Delta_{2}(T)= & \int_{0}^{T} q\left[\pi_{t}(X)-\pi_{t}(\bar{X})\right]\left[\bar{X}_{t}-\pi_{t}(\bar{X})\right] \mathrm{d} t+\bar{\rho} \int_{0}^{T}\left[\pi_{t}(X)-\pi_{t}(\bar{X})\right] \gamma_{c}^{12}(t, t) \mathrm{d} \nu_{t} \\
& -\int_{0}^{T} p_{t} d\left[\pi_{t}(X)-\pi_{t}(\bar{X})\right]-\int_{0}^{T}\left[\pi_{t}(X)-\pi_{t}(\bar{X})\right] \mathrm{d} p_{t} .
\end{aligned}
$$

Notice that, integrating by parts in the last line above, since $\pi_{0}(X)-\pi_{0}(\bar{X})=0$, we can rewrite $\Delta_{2}(T)$ as

$$
\begin{aligned}
\Delta_{2}(T)= & \int_{0}^{T} q\left[\pi_{t}(X)-\pi_{t}(\bar{X})\right]\left[\bar{X}_{t}-\pi_{t}(\bar{X})\right] \mathrm{d} t-p_{T}\left[\pi_{T}(X)-\pi_{T}(\bar{X})\right] \\
& +\bar{\rho} \int_{0}^{T}\left[\pi_{t}(X)-\pi_{t}(\bar{X})\right] \gamma_{c}^{12}(t, t) \mathrm{d} \nu_{t}
\end{aligned}
$$

Since $J_{\infty}(u)=\lim \sup _{T \rightarrow+\infty} \frac{1}{T} j_{T}(u)$ a.s. and $\Delta_{1}(T) \geq 0$, of course we have

$$
J_{\infty}(u) \geq J_{\infty}(\bar{u})+\liminf _{T \rightarrow+\infty} \frac{1}{T} \Delta_{2}(T) \quad \text { a.s. }
$$

Hence, in order to prove that $\bar{u}$ minimizes $J_{\infty}$ over $\mathcal{U}_{a d}$, it is sufficient to show that $\lim _{T \rightarrow+\infty} \frac{1}{T} \Delta_{2}(T)=0$ a.s. To this end, it suffices to show that, if $u \in \mathcal{U}_{a d}$ is such that $J_{\infty}(u)<+\infty$, then the following limits hold a.s.

$$
\begin{gathered}
\lim _{T \rightarrow+\infty} \frac{1}{T} \int_{0}^{T}\left[\pi_{t}(X)-\pi_{t}(\bar{X})\right]\left[\bar{X}_{t}-\pi_{t}(\bar{X})\right] \mathrm{d} t=0, \\
\lim _{T \rightarrow+\infty} \frac{1}{T} p_{T}\left[\pi_{T}(X)-\pi_{T}(\bar{X})\right]=0
\end{gathered}
$$

and

$$
\lim _{T \rightarrow+\infty} \frac{1}{T} \int_{0}^{T}\left[\pi_{t}(X)-\pi_{t}(\bar{X})\right] \gamma_{c}^{12}(t, t) \mathrm{d} \nu_{t}=0 .
$$

These three properties will be proved in the Appendix (cf. Sect. 6.3).

\section{INFINITE TIME HORIZON CONTROL PROBlEM - SECOND SOLUtion}

Here the setting is the same as in Section 4. At first, it is worth emphasizing that the analysis therein has provided not only an optimal control but also the explicit value of the minimal cost. Hence for any other control possibly candidate for optimality, the only thing to check is that it achieves the minimal cost. On this basis, now we propose a simpler and more explicit control which is also optimal. With respect to the first solution, it has the advantage that the involved feedback is more clearly expressed in terms of the observation process $Y$. Moreover, it is coherent with the asymptotically optimal filter proposed in [11] for linear systems without control and with the second solution proposed in [12] for the infinite time horizon optimal control problem under complete observation. 
In the classical case $H=\frac{1}{2}$ where the noises are standard Brownian motions and hence the system of filtering equations reduces to the usual Kalman-Bucy system, the asymptotic variance of the filtering error is $\bar{\gamma}$ given by (11). In that case, substituting the constant $\bar{\gamma}$ for the function $\gamma(t)$ in the equations (5) and (9), one gets the simpler controller

$$
\left\{\begin{aligned}
u_{t}^{*} & =-\frac{b}{r} \bar{\rho} \pi_{t}^{*} ; \quad X_{t}^{*}=X_{t}^{u^{*}} ; \quad Y_{t}^{*}=Y_{t}^{u^{*}}, \\
\mathrm{~d} \pi_{t}^{*} & =\left[a \pi_{t}^{*}+b u_{t}^{*}\right] \mathrm{d} t+\frac{A}{B^{2}} \bar{\gamma}\left[\mathrm{d} Y_{t}^{*}-A \pi_{t}^{*} \mathrm{~d} t\right], \quad \pi_{0}^{*}=0,
\end{aligned}\right.
$$

where $\bar{\rho}$ is still given by (8). Observe that, taking into account (8) and (11), actually $\pi_{t}^{*}$ is governed by

$$
\mathrm{d} \pi_{t}^{*}=-\left[a+\delta_{c}+\delta_{f}\right] \pi_{t}^{*} \mathrm{~d} t+\frac{A}{B^{2}} \bar{\gamma} \mathrm{d} Y_{t}^{*} ; \pi_{0}^{*}=0
$$

Hence $\pi_{t}^{*}$ can be represented as

$$
\pi_{t}^{*}=\frac{A}{B^{2}} \bar{\gamma} \int_{0}^{t} \mathrm{e}^{-\left[a+\delta_{c}+\delta_{f}\right](t-s)} \mathrm{d} Y_{s}^{*} .
$$

It can be checked that, as a filter of $X_{t}^{*}$ from the observations $\left\{Y_{s}^{*}, s \leq t\right\}, \pi_{t}^{*}$ is asymptotically optimal in the sense that, as $t$ goes to infinity, the variance $\mathbb{E}\left(X_{t}^{*}-\pi_{t}^{*}\right)^{2}$ of the corresponding filtering error converges to the same limit $\bar{\gamma}$ as $\mathbb{E}\left(X_{t}^{*}-\pi_{t}\left(X^{*}\right)\right)^{2}$. Moreover, it can also be checked that the triple $\left(u^{*}, X^{*}, Y^{*}\right)$ is actually optimal in the infinite time horizon control problem, i.e., $J_{\infty}\left(u^{*}\right)=J_{\infty}(\bar{u})$ given by (10). Observe that, in this case, the optimality is achieved in the class of controls which can be represented as $\int_{0}^{t} \phi(t-s) \mathrm{d} Y_{s}$. Below, we show that this still holds for $H>\frac{1}{2}$ and we identify in this class a control for which the cost value is $J_{\infty}(\bar{u})$ given by (45).

So, we start with a control $u_{t}=u_{t}^{\phi}$ in the form

$$
u_{t}=\int_{0}^{t} \phi(t-s) \mathrm{d} Y_{s}
$$

Then, from (1), it is readily seen that we have also ${ }^{1}$

$$
\left\{\begin{array}{l}
u_{t}=\int_{0}^{t} \mathcal{U}_{V}(t-s) \mathrm{d} V_{s}^{H}+\int_{0}^{t} \mathcal{U}_{W}(t-s) \mathrm{d} W_{s}^{H} \\
X_{t}=\int_{0}^{t} \mathcal{X}_{V}(t-s) \mathrm{d} V_{s}^{H}+\int_{0}^{t} \mathcal{X}_{W}(t-s) \mathrm{d} W_{s}^{H}
\end{array}\right.
$$

where

$$
\left\{\begin{array}{l}
\dot{\mathcal{X}}_{V}(\tau)=a \mathcal{X}_{V}(\tau)+b \mathcal{U}_{V}(\tau), \tau \geq 0 ; \mathcal{X}_{V}(0)=\sigma \\
\dot{\mathcal{X}}_{W}(\tau)=a \mathcal{X}_{W}(\tau)+b \mathcal{U}_{W}(\tau), \tau \geq 0 ; \mathcal{X}_{W}(0)=0, \\
\mathcal{U}_{V}(\tau)=A \int_{0}^{\tau} \phi(v) \mathcal{X}_{V}(\tau-v) \mathrm{d} v, \tau \geq 0 \\
\mathcal{U}_{W}(\tau)=A \int_{0}^{\tau} \phi(v) \mathcal{X}_{W}(\tau-v) \mathrm{d} v+B \phi(\tau), \tau \geq 0
\end{array}\right.
$$

\footnotetext{
${ }^{1}$ For simplicity, here we deal only with the case $x=0$.
} 
Actually, it can be recognized that $\mathcal{X}_{W} \equiv(B b / A \sigma) \mathcal{U}_{V}$. Hence, for the system (62)-(63), we can substitute the following:

$$
\left\{\begin{array}{l}
u_{t}=\int_{0}^{t} \mathcal{U}_{V}(t-s) \mathrm{d} V_{s}^{H}+\int_{0}^{t} \mathcal{U}_{W}(t-s) \mathrm{d} W_{s}^{H}, \\
X_{t}=\int_{0}^{t} \mathcal{X}_{V}(t-s) \mathrm{d} V_{s}^{H}+\frac{B b}{A \sigma} \int_{0}^{t} \mathcal{U}_{V}(t-s) \mathrm{d} W_{s}^{H},
\end{array}\right.
$$

where

$$
\left\{\begin{array}{l}
\dot{\mathcal{X}}_{V}(\tau)=a \mathcal{X}_{V}(\tau)+b \mathcal{U}_{V}(\tau), \tau \geq 0 ; \mathcal{X}_{V}(0)=\sigma \\
\dot{\mathcal{U}}_{V}(\tau)=a \mathcal{U}_{V}(\tau)+\frac{A \sigma}{B} \mathcal{U}_{W}(\tau), \tau \geq 0 ; \mathcal{U}_{V}(0)=0
\end{array}\right.
$$

and

$$
\mathcal{U}_{W}(\tau)=\frac{B b}{\sigma} \int_{0}^{\tau} \phi(v) \mathcal{U}_{V}(\tau-v) \mathrm{d} v+B \phi(\tau), \tau \geq 0
$$

We observe that in the system defined by the last two equations above which link $\phi, \mathcal{U}_{V}$ and $\mathcal{U}_{W}$, to fix a function $\phi$ is equivalent to fix a function $\mathcal{U}_{W}$. So, we may forget about the last equation and from now on, instead of a function $\phi$, we look for an appropriate function $\mathcal{U}_{W}$. Of course, we are interested in those functions $\mathcal{U}_{W}$ such that, for the process $(u, X)$ given by $(64)-(65)$, the $\operatorname{limits} \lim _{T \rightarrow+\infty} T^{-1} \int_{0}^{T} u_{t}^{2} \mathrm{~d} t$ and $\lim _{T \rightarrow+\infty} T^{-1} \int_{0}^{T} X_{t}^{2} \mathrm{~d} t$ exist and are finite almost surely. Our guess is that the minimum for $J_{\infty}$ can be obtained by choosing $\mathcal{U}_{W}$ in such a way that these limits are nothing but $\lim _{T \rightarrow+\infty} \mathbb{E} u_{T}^{2}$ and $\lim _{T \rightarrow+\infty} \mathbb{E} X_{T}^{2}$ respectively and moreover the minimum value of

$$
\hat{J}_{\infty}\left(\mathcal{U}_{W}\right)=\lim _{T \rightarrow+\infty} \mathbb{E}\left[q X_{T}^{2}+r u_{T}^{2}\right]
$$

is achieved. Actually, for a stochastic integral

$$
S_{t}=\int_{0}^{t} g(t-s) \mathrm{d} B_{s}^{H}
$$

we can evaluate

$$
\lim _{T \rightarrow+\infty} \mathbb{E} S_{T}^{2}=H(2 H-1) \int_{0}^{+\infty} \int_{0}^{+\infty} g(s) g(r)|s-r|^{2 H-2} \mathrm{~d} s \mathrm{~d} r .
$$

Exploiting the representation

$$
|s-r|^{2 H-2}=\frac{1}{B\left(H-\frac{1}{2}, 2-2 H\right)} \int_{s \vee r}^{+\infty}(\tau-s)^{H-\frac{3}{2}}(\tau-r)^{H-\frac{3}{2}} \mathrm{~d} \tau,
$$

it is easy to check that we can rewrite

$$
\lim _{T \rightarrow+\infty} \mathbb{E} S_{T}^{2}=\frac{2 H \Gamma\left(\frac{3}{2}-H\right)}{\Gamma\left(H+\frac{1}{2}\right) \Gamma(2-2 H)} \int_{0}^{+\infty} \widetilde{g}^{2}(s) \mathrm{d} s
$$

where

$$
\widetilde{g}(s)=\frac{d}{d s} \int_{0}^{s} g(r)(s-r)^{H-\frac{1}{2}} \mathrm{~d} r .
$$

Hence, due to $(64)$ and the independence between $V^{H}$ and $W^{H}$, we can rewrite the quantity $\hat{J}_{\infty}\left(\mathcal{U}_{W}\right)$ as

$$
\widetilde{J}_{\infty}\left(\widetilde{\mathcal{U}}_{W}\right)=\frac{2 H \Gamma\left(\frac{3}{2}-H\right)}{\Gamma\left(H+\frac{1}{2}\right) \Gamma(2-2 H)} \int_{0}^{+\infty}\left\{q \widetilde{\mathcal{X}}_{V}^{2}(s)+\left[q \frac{B^{2} b^{2}}{A^{2} \sigma^{2}}+r\right] \widetilde{\mathcal{U}}_{V}^{2}(s)+r \widetilde{\mathcal{U}}_{W}^{2}(s)\right\} \mathrm{d} s
$$


where the triple $\left(\widetilde{\mathcal{X}}_{V}, \widetilde{\mathcal{U}}_{V}, \widetilde{\mathcal{U}}_{W}\right)$ corresponds to $\left(\mathcal{X}_{V}, \mathcal{U}_{V}, \mathcal{U}_{W}\right)$ by $(67)$. Actually, it can be readily seen from (65) that the dynamics which link $\widetilde{\mathcal{X}}_{V}, \widetilde{\mathcal{U}}_{V}, \widetilde{\mathcal{U}}_{W}$ are nothing but

$$
\left\{\begin{array}{l}
\dot{\tilde{\mathcal{X}}}_{V}(\tau)=a \widetilde{\mathcal{X}}_{V}(\tau)+b \widetilde{\mathcal{U}}_{V}(\tau)+\sigma\left(H-\frac{1}{2}\right) \tau^{H-\frac{3}{2}}, \tau \geq 0 ; \widetilde{\mathcal{X}}_{V}(0)=0, \\
\dot{\widetilde{\mathcal{U}}}_{V}(\tau)=a \widetilde{\mathcal{U}}_{V}(\tau)+\frac{A \sigma}{B} \widetilde{\mathcal{U}}_{W}(\tau), \tau \geq 0 ; \widetilde{\mathcal{U}}_{V}(0)=0 .
\end{array}\right.
$$

Now we solve the infinite time horizon deterministic control problem.

$$
\min _{\widetilde{\mathcal{U}}_{W}} \widetilde{J}_{\infty}\left(\widetilde{\mathcal{U}}_{W}\right) \quad \text { subject to (69). }
$$

Let us define the $2 \times 2$ matrices $\mathcal{A}, \mathcal{Q}$ and the vector $\mathcal{B}$ in $\mathbb{R}^{2}$ by:

$$
\mathcal{A}=\left(\begin{array}{cc}
a & b \\
0 & a
\end{array}\right) ; \quad \mathcal{Q}=\left(\begin{array}{cc}
q & 0 \\
0 & q \frac{B^{2} b^{2}}{A^{2} \sigma^{2}}+r
\end{array}\right) ; \quad \mathcal{B}=\left(\begin{array}{c}
0 \\
\frac{A \sigma}{B}
\end{array}\right)
$$

and introduce also the $\mathbb{R}^{2}$-valued functions $\widetilde{\mathcal{Z}}_{V}(\tau)$ and $\mathcal{K}(\tau)$ as

$$
\widetilde{\mathcal{Z}}_{V}(\tau)=\left(\begin{array}{c}
\widetilde{\mathcal{X}}_{V}(\tau) \\
\widetilde{\mathcal{U}}_{V}(\tau)
\end{array}\right) ; \quad \mathcal{K}(\tau)=\left(\begin{array}{c}
\sigma\left(H-\frac{1}{2}\right) \tau^{H-\frac{3}{2}} \\
0
\end{array}\right)
$$

Then, we can rewrite (69) and (68) as

$$
\dot{\mathcal{Z}}_{V}(\tau)=\mathcal{A} \widetilde{\mathcal{Z}}_{V}(\tau)+\mathcal{B} \tilde{\mathcal{U}}_{W}(\tau)+\mathcal{K}(\tau), \tau \geq 0 ; \widetilde{\mathcal{Z}}_{V}(0)=0
$$

and

$$
\widetilde{J}_{\infty}\left(\widetilde{\mathcal{U}}_{W}\right)=\frac{2 H \Gamma\left(\frac{3}{2}-H\right)}{\Gamma\left(H+\frac{1}{2}\right) \Gamma(2-2 H)} \int_{0}^{+\infty}\left\{\widetilde{\mathcal{Z}}_{V}^{\prime}(s) \mathcal{Q} \widetilde{\mathcal{Z}}_{V}(s)+r \widetilde{\mathcal{U}}_{W}^{2}(s)\right\} \mathrm{d} s .
$$

Hence, to solve the problem (70), it is natural to introduce the following Hamiltonian system in the functions $\widetilde{\mathcal{Z}}^{*}$ and $\widetilde{p}^{*}$ :

$$
\left\{\begin{array}{l}
\dot{\widetilde{\mathcal{Z}}}^{*}(\tau)=\mathcal{A} \widetilde{\mathcal{Z}}^{*}(\tau)-r^{-1} \mathcal{B} \mathcal{B}^{\prime} \widetilde{p}^{*}(\tau)+\mathcal{K}(\tau), \widetilde{\mathcal{Z}}^{*}(0)=0 ; \lim _{\tau \rightarrow+\infty} \widetilde{\mathcal{Z}}^{*}(\tau)=0, \\
\dot{\tilde{p}}^{*}(\tau)=-\mathcal{Q} \widetilde{\mathcal{Z}}^{*}(\tau)-\mathcal{A}^{\prime} \widetilde{p}^{*}(\tau) ; \lim _{\tau \rightarrow+\infty} \widetilde{p}^{*}(\tau)=0 .
\end{array}\right.
$$

Observe that, if $\left(\widetilde{\mathcal{Z}}^{*}, \widetilde{p}^{*}\right)$ is a solution of $(73)$, then $\widetilde{\mathcal{Z}}^{*}=\left(\widetilde{\mathcal{X}}_{V}^{*}, \widetilde{\mathcal{U}}_{V}^{*}\right)^{\prime}$ is nothing but the solution of (71) corresponding to the control

$$
\widetilde{\mathcal{U}}_{W}^{*}(\tau)=-r^{-1} \mathcal{B}^{\prime} \widetilde{p}^{*}(\tau)
$$

Actually, standard calculations permit to show that if $\left(\widetilde{\mathcal{Z}}^{*}, \widetilde{p}^{*}\right)$ is a solution of $(73)$, then the pair $\left(\widetilde{\mathcal{Z}}^{*}, \widetilde{\mathcal{U}}_{W}^{*}\right)$ is an optimal pair in the control problem (70). So, finally, to solve this problem we have just to identify a solution of (73). Let us look for a solution in such a way that for some fixed $2 \times 2$ matrix $\Gamma$ and $\mathbb{R}^{2}$-valued function $\tilde{\lambda}^{*}$, the function $\widetilde{p}^{*}$ can be represented as

$$
\widetilde{p}^{*}(\tau)=\Gamma\left[\widetilde{\mathcal{Z}}^{*}(\tau)+\widetilde{\lambda}^{*}(\tau)\right]
$$

Hence, it is readily seen that one may take $\Gamma$ as the nonnegative solution of the algebraic Riccati equation

$$
\mathcal{A}^{\prime} \Gamma+\Gamma \mathcal{A}-r^{-1} \Gamma \mathcal{B} \mathcal{B}^{\prime} \Gamma+\mathcal{Q}=0,
$$


and $\widetilde{\lambda}^{*}$ as the solution of the differential equation

$$
\Gamma \tilde{\vec{\lambda}}^{*}(\tau)=\left[r^{-1} \Gamma \mathcal{B} \mathcal{B}^{\prime}-\mathcal{A}^{\prime}\right] \Gamma \tilde{\lambda}^{*}(\tau)-\Gamma \mathcal{K}(\tau) ; \lim _{\tau \rightarrow+\infty} \tilde{\lambda}^{*}(\tau)=0
$$

It can be checked that the matrices $\Gamma$ and $\Lambda=r^{-1} \Gamma \mathcal{B} \mathcal{B}^{\prime}-\mathcal{A}^{\prime}$ are given by

$$
\Gamma=\frac{B^{2} r}{A^{2} \sigma^{2} b^{2}}\left(\begin{array}{cc}
\left(a+\delta_{c}\right)\left(a+\delta_{f}\right)\left(\delta_{c}+\delta_{f}\right) & b\left(a+\delta_{c}\right)\left(a+\delta_{f}\right) \\
b\left(a+\delta_{c}\right)\left(a+\delta_{f}\right) & b^{2}\left(2 a+\delta_{c}+\delta_{f}\right)
\end{array}\right),
$$

and

$$
\Lambda=r^{-1} \Gamma \mathcal{B B}^{\prime}-\mathcal{A}^{\prime}=\left(\begin{array}{cc}
-a & b^{-1}\left(a+\delta_{c}\right)\left(a+\delta_{f}\right) \\
-b & a+\delta_{c}+\delta_{f}
\end{array}\right) .
$$

The eigenvalues of $\Lambda$ are $\delta_{c}$ and $\delta_{f}$. If $\delta_{c} \neq \delta_{f}$, then it can be decomposed as

$$
\Lambda=P_{-}\left(\begin{array}{cc}
\delta_{c} & 0 \\
0 & \delta_{f}
\end{array}\right) P_{-}^{-1}
$$

where

Similarly, if $\delta_{c}=\delta_{f}$, then $\Lambda$ can be decomposed as

$$
P_{-}=\left(\begin{array}{cc}
a+\delta_{f} & a+\delta_{c} \\
b & b
\end{array}\right) ; \quad P_{-}^{-1}=\frac{1}{b\left(\delta_{f}-\delta_{c}\right)}\left(\begin{array}{cc}
b & -\left(a+\delta_{c}\right) \\
-b & a+\delta_{f}
\end{array}\right) .
$$

$$
\Lambda=P_{+}\left(\begin{array}{cc}
\delta_{c} & \frac{1}{b}\left[b^{2}+\left(a+\delta_{c}\right)^{2}\right] \\
0 & \delta_{c}
\end{array}\right) P_{+}^{-1}
$$

where

Of course, we have

$$
P_{+}=\left(\begin{array}{cc}
a+\delta_{c} & -b \\
b & a+\delta_{c}
\end{array}\right) ; \quad P_{+}^{-1}=\frac{1}{b^{2}+\left(a+\delta_{c}\right)^{2}}\left(\begin{array}{cc}
a+\delta_{c} & b \\
-b & a+\delta_{c}
\end{array}\right)
$$

or

$$
\Gamma \tilde{\lambda}^{*}(t)=\mathrm{e}^{\Lambda t} \int_{t}^{+\infty} \mathrm{e}^{-\Lambda s} \Gamma \mathcal{K}(s) \mathrm{d} s
$$

For $\delta_{c} \neq \delta_{f}$, this gives

$$
\Gamma \widetilde{\lambda}^{*}(t)=\int_{0}^{+\infty} \mathrm{e}^{-\Lambda \tau} \Gamma \mathcal{K}(t+\tau) \mathrm{d} \tau \text {. }
$$

$$
\Gamma \widetilde{\lambda}^{*}(t)=\frac{\bar{\rho} \bar{\gamma}}{\sigma\left(\delta_{f}-\delta_{c}\right)}\left(\begin{array}{c}
\left(\delta_{f}^{2}-a^{2}\right) \widetilde{\varphi}_{c}(t)-\left(\delta_{c}^{2}-a^{2}\right) \widetilde{\varphi}_{f}(t) \\
b\left[\left(\delta_{f}-a\right) \widetilde{\varphi}_{c}(t)-\left(\delta_{c}-a\right) \widetilde{\varphi}_{f}(t)\right]
\end{array}\right)
$$

where

$$
\widetilde{\varphi}_{f}(t)=\mathrm{e}^{\delta_{f} t} \int_{t}^{+\infty} \mathrm{e}^{-\delta_{f} s} \mathrm{~d} s^{H-\frac{1}{2}} ; \quad \widetilde{\varphi}_{c}(t)=\mathrm{e}^{\delta_{c} t} \int_{t}^{+\infty} \mathrm{e}^{-\delta_{c} s} \mathrm{~d} s^{H-\frac{1}{2}},
$$

and in particular

$$
\Gamma \widetilde{\lambda}^{*}(0)=\frac{\Gamma\left(H+\frac{1}{2}\right)}{\sigma} \frac{\bar{\rho} \bar{\gamma}}{\delta_{f}-\delta_{c}}\left(\begin{array}{c}
\left(\delta_{f}^{2}-a^{2}\right) \delta_{c}^{\frac{1}{2}-H}-\left(\delta_{c}^{2}-a^{2}\right) \delta_{f}^{\frac{1}{2}-H} \\
b\left[\left(\delta_{f}-a\right) \delta_{c}^{\frac{1}{2}-H}-\left(\delta_{c}-a\right) \delta_{f}^{\frac{1}{2}-H}\right]
\end{array}\right) .
$$

Concerning the cost, starting from $(73)$ and evaluating by integration by parts the variation of $\widetilde{p}^{*}(t)^{\prime} \widetilde{\mathcal{Z}}^{*}(t)$ over $[0,+\infty)$, it is readily seen that

$$
\widetilde{J}_{\infty}\left(\widetilde{\mathcal{U}}_{W}^{*}\right)=\frac{2 H \Gamma\left(\frac{3}{2}-H\right)}{\Gamma\left(H+\frac{1}{2}\right) \Gamma(2-2 H)} \int_{0}^{+\infty} \mathcal{K}^{\prime}(\tau) \Gamma \widetilde{p}^{*}(\tau) \mathrm{d} \tau
$$


Consequently, evaluating by integration by parts the variation of $\widetilde{\lambda}^{*}(t)^{\prime} \Gamma \widetilde{\mathcal{Z}}^{*}(t)$ over $[0,+\infty)$, we get that $\widetilde{J}_{\infty}\left(\widetilde{\mathcal{U}}_{W}^{*}\right)$ can also be represented as

$$
\widetilde{J}_{\infty}\left(\widetilde{\mathcal{U}}_{W}^{*}\right)=\frac{2 H \Gamma\left(\frac{3}{2}-H\right)}{\Gamma\left(H+\frac{1}{2}\right) \Gamma(2-2 H)}\left\{2 \int_{0}^{+\infty} \mathcal{K}^{\prime}(\tau) \Gamma \widetilde{\lambda}^{*}(\tau) \mathrm{d} \tau-r^{-1} \int_{0}^{+\infty} \widetilde{\lambda}^{*}(\tau)^{\prime} \Gamma \mathcal{B} \mathcal{B}^{\prime} \Gamma \widetilde{\lambda}^{*}(\tau) \mathrm{d} \tau\right\} .
$$

Then, evaluating the variation of $\widetilde{\lambda}^{*}(t)^{\prime} \Gamma \widetilde{\lambda}^{*}(t)$ over $[0,+\infty)$, it comes that also

$$
\widetilde{J}_{\infty}\left(\widetilde{\mathcal{U}}_{W}^{*}\right)=\frac{2 H \Gamma\left(\frac{3}{2}-H\right)}{\Gamma\left(H+\frac{1}{2}\right) \Gamma(2-2 H)}\left\{\widetilde{\lambda}^{*}(0)^{\prime} \Gamma \widetilde{\lambda}^{*}(0)+\int_{0}^{+\infty} \widetilde{\lambda}^{*}(\tau)^{\prime} \mathcal{Q} \widetilde{\lambda}^{*}(\tau) \mathrm{d} \tau\right\}
$$

Finally, an alternative expression for $\widetilde{J}_{\infty}\left(\widetilde{\mathcal{U}}_{W}^{*}\right)$ can be derived. We introduce the nonnegative solution $R$ of the algebraic Lyapunov equation

$$
\Lambda^{\prime} R+R \Lambda=r^{-1} \mathcal{B B}^{\prime} .
$$

Then, evaluating by integration by parts the variation of $\widetilde{\lambda}^{*}(t)^{\prime} \Gamma R \Gamma \widetilde{\lambda}^{*}(t)$ over $[0,+\infty)$, it comes that we can also write

$$
\widetilde{J}_{\infty}\left(\widetilde{\mathcal{U}}_{W}^{*}\right)=\frac{2 H \Gamma\left(\frac{3}{2}-H\right)}{\Gamma\left(H+\frac{1}{2}\right) \Gamma(2-2 H)}\left\{\widetilde{\lambda}^{*}(0)^{\prime} \Gamma R \Gamma \widetilde{\lambda}^{*}(0)+2 \int_{0}^{+\infty} \mathcal{K}^{\prime}(\tau)[I-\Gamma R] \Gamma \widetilde{\lambda}^{*}(\tau) \mathrm{d} \tau\right\}
$$

Actually, we get

$$
R=\frac{A^{2} \sigma^{2}}{2 B^{2} r} \frac{1}{\delta_{c} \delta_{f}\left(\delta_{c}+\delta_{f}\right)}\left(\begin{array}{cc}
b^{2} & -a b \\
-a b & a^{2}+\delta_{c} \delta_{f}
\end{array}\right) .
$$

Now, from (78)-(80), we can compute the value $\widetilde{J}_{\infty}\left(\widetilde{\mathcal{U}}_{W}^{*}\right)$. At first, from (78), it comes that the second term within brackets in the right hand side of (81) gives

$$
q \sigma^{2} \frac{\Gamma\left(H+\frac{1}{2}\right) \Gamma(2-2 H) \Gamma(2 H)}{2 \Gamma\left(\frac{3}{2}-H\right)} \frac{\left(\delta_{f}^{2}-a^{2}\right) \delta_{c}^{-2 H}-\left(\delta_{c}^{2}-a^{2}\right) \delta_{f}^{-2 H}}{\delta_{f}^{2}-\delta_{c}^{2}} .
$$

Moreover, from (80), it comes that the first term within brackets in the right hand side of (81) gives

$$
\begin{gathered}
\Gamma^{2}\left(H+\frac{1}{2}\right) \frac{A^{2} \bar{\gamma}^{2} \bar{\rho}}{2 B^{2}} \frac{\delta_{c}+a}{\delta_{c} \delta_{f}\left(\delta_{f}-\delta_{c}\right)^{2}\left(\delta_{f}+\delta_{c}\right)}\left\{\left[\delta_{c}^{\frac{1}{2}-H} \delta_{f}\left(\delta_{f}-a\right)-\delta_{f}^{\frac{1}{2}-H} \delta_{c}\left(\delta_{c}-a\right)\right]^{2}\right. \\
\left.+\delta_{c} \delta_{f}\left[\delta_{c}^{\frac{1}{2}-H}\left(\delta_{f}-a\right)-\delta_{f}^{\frac{1}{2}-H}\left(\delta_{c}-a\right)\right]^{2}\right\} .
\end{gathered}
$$

Finally, from these expressions, it can be checked that $\widetilde{J}_{\infty}\left(\widetilde{\mathcal{U}}_{W}^{*}\right)$ can be represented as

$$
\widetilde{J}_{\infty}\left(\widetilde{\mathcal{U}}_{W}^{*}\right)=\zeta_{\infty}(H)+q \gamma_{\infty}(H),
$$

where $\zeta_{\infty}(H)$ and $\gamma_{\infty}(H)$ are given by $(46)$ and (47), respectively. In other words, $\widetilde{J}_{\infty}\left(\widetilde{\mathcal{U}}_{W}^{*}\right)$ is nothing but the optimal cost $J_{\infty}(\bar{u})$ given by (45) for our original infinite horizon stochastic control problem.

The case $\delta_{c}=\delta_{f}$ can be treated similarly to obtain the same conclusion, with the limiting value (48) instead of (46) for the term $\zeta_{\infty}(H)$.

Now, taking into account the fact that the connection (67) can be inverted by

$$
g(s)=\frac{1}{B\left(H+\frac{1}{2}, \frac{3}{2}-H\right)} \frac{\mathrm{d}}{\mathrm{d} s} \int_{0}^{s} \widetilde{g}(r)(s-r)^{\frac{1}{2}-H} \mathrm{~d} r
$$


we may reformulate our initial guess by telling that the triple $\left(\mathcal{U}_{V}^{*}, \mathcal{X}_{V}^{*}, \mathcal{U}_{W}^{*}\right)$, obtained from $\left(\widetilde{\mathcal{U}}_{V}^{*}, \widetilde{\mathcal{X}}_{V}^{*}, \widetilde{\mathcal{U}}_{W}^{*}\right)$ through (82), is a candidate to define through (64) an optimal pair $\left(u^{*}, X^{*}\right)$ in the original infinite horizon stochastic control problem. Actually the proof that this is true is included below in the proof of the following statement.

Theorem 4.1. Let the pairs of constants $\left(\bar{\rho}, \delta_{c}\right)$ and $\left(\bar{\gamma}, \delta_{f}\right)$ be defined by (8) and (11) respectively. In the control problem

$$
\min _{u \in \mathcal{U}_{a d}} J_{\infty}(u) \text { subject to }(1)
$$

with $J_{\infty}$ defined by $(7)$, an optimal control $u^{*}$ in $\mathcal{U}_{a d}$ and the corresponding triple $\left(u^{*}, X^{*}, Y^{*}\right)$ are governed by the system

$$
u_{t}^{*}=-\frac{b}{r} \bar{\rho}\left[\pi_{t}^{*}+\frac{A \sigma^{2}}{B} \frac{v_{t}^{f}-v_{t}^{c}}{\delta_{c}-\delta_{f}}\right] ; \quad X_{t}^{*}=X_{t}^{u^{*}} ; \quad Y_{t}^{*}=Y_{t}^{u^{*}},
$$

where the process $\pi^{*}$ is defined by

$$
\pi_{t}^{*}=\hat{\pi}_{t}+\frac{A}{B} \bar{\gamma} v_{t}^{f}
$$

with

$$
\mathrm{d} \hat{\pi}_{t}=a \hat{\pi}_{t} \mathrm{~d} t+b u_{t}^{*} \mathrm{~d} t+\frac{A}{B^{2}} \bar{\gamma}\left[\mathrm{d} Y_{t}^{*}-A \hat{\pi}_{t} \mathrm{~d} t\right] ; \quad \hat{\pi}_{0}=x,
$$

and the processes $v^{c}$ and $v^{f}$ satisfy the integral equations

$$
\begin{aligned}
& v_{t}^{c}=\int_{0}^{t} \delta_{c} v_{s}^{c} \mathrm{~d} s+\int_{0}^{t}\left[\frac{\delta_{c}^{\frac{1}{2}-H}}{\Gamma\left(\frac{3}{2}-H\right)}(t-s)^{\frac{1}{2}-H}-1\right] B^{-1}\left[\mathrm{~d} Y_{s}^{*}-A \hat{\pi}_{s} \mathrm{~d} s\right], \\
& v_{t}^{f}=\int_{0}^{t} \delta_{f} v_{s}^{f} \mathrm{~d} s+\int_{0}^{t}\left[\frac{\delta_{f}^{\frac{1}{2}-H}}{\Gamma\left(\frac{3}{2}-H\right)}(t-s)^{\frac{1}{2}-H}-1\right] B^{-1}\left[\mathrm{~d} Y_{s}^{*}-A \hat{\pi}_{s} \mathrm{~d} s\right] .
\end{aligned}
$$

Remark 4.2. (a) Actually, in the statement above, for $\delta_{f}=\delta_{c}$, one has $v^{f} \equiv v^{c}$. The term $\left(v^{c}-v^{f}\right) /\left(\delta_{c}-\delta_{f}\right)$ must be changed into a process $\dot{v}^{c}$ which satisfies the equation

$$
\dot{v}_{t}^{c}=\int_{0}^{t}\left[v_{s}^{c}+\delta_{c} \dot{v}_{s}^{c}\right] \mathrm{d} s+\int_{0}^{t}\left(\frac{1}{2}-H\right) \frac{\delta_{c}^{-\frac{1}{2}-H}}{\Gamma\left(\frac{3}{2}-H\right)}(t-s)^{\frac{1}{2}-H} B^{-1}\left[\mathrm{~d} Y_{s}^{*}-A \hat{\pi}_{s} \mathrm{~d} s\right] .
$$

(b) In the case $H=1 / 2$, one has $v^{f} \equiv v^{c} \equiv 0$ and hence it is readily seen that the statement reduces globally to the well known result recalled at the beginning of Section 5 .

(c) As a filter of $X_{t}^{*}$ from the observations $\left\{Y_{s}^{*}, s \leq t\right\}, \pi_{t}^{*}$ defined by (84) is asymptotically optimal in the sense that, as $t$ goes to infinity, the variance $\mathbb{E}\left(X_{t}^{*}-\pi_{t}^{*}\right)^{2}$ of the corresponding filtering error converges to the same limit $\gamma_{\infty}(H)$ given by $(47)$ as $\mathbb{E}\left(X_{t}^{*}-\pi_{t}\left(X^{*}\right)\right)^{2}$ for the optimal filter $\pi_{t}\left(X^{*}\right)$. This can be seen by reworking the developments in [11] concerning an autonomous linear system with $u \equiv 0$, which generates the pair $\left(X^{0}, Y^{0}\right)$. The result therein can be reformulated as follows: defining the processes

$$
\mathrm{d} \pi_{t}^{0}=a \pi_{t}^{0} \mathrm{~d} t+\frac{A}{B^{2}} \bar{\gamma}\left[\mathrm{d} Y_{t}^{0}-A \pi_{t}^{0} \mathrm{~d} t\right] ; \quad \pi_{0}^{0}=0
$$

and

$$
v_{t}^{0}=\int_{0}^{t} \delta_{f} v_{s}^{0} \mathrm{~d} s+\int_{0}^{t}\left[\frac{\delta_{f}^{\frac{1}{2}-H}}{\Gamma\left(\frac{3}{2}-H\right)}(t-s)^{\frac{1}{2}-H}-1\right] B^{-1}\left[\mathrm{~d} Y_{s}^{0}-A \pi_{s}^{0} \mathrm{~d} s\right],
$$

then $\pi_{t}^{0}+\frac{A}{B} \bar{\gamma} v_{t}^{0}$ is asymptotically optimal as a filter of $X_{t}^{0}$ from the observations $\left\{Y_{s}^{0}, s \leq t\right\}$. Now, observing that actually $X^{*}-\hat{\pi} \equiv X^{0}-\pi^{0}, Y^{*}-\int_{0}^{\cdot} A \hat{\pi} \mathrm{d} s \equiv Y^{0}-\int_{0} A \pi^{0} \mathrm{~d} s$ and hence also $v^{f} \equiv v^{0}$, it follows that $\pi_{t}^{*}=\hat{\pi}_{t}+\frac{A}{B} \bar{\gamma} v_{t}^{f}$ is also asymptotically optimal as a filter of $X_{t}^{*}$ from the observations $\left\{Y_{s}^{*}, s \leq t\right\}$. 
Proof of Theorem 4.1. The statement for arbitrary values of $\sigma$ and $B$ can be easily derived from the statement for $\sigma=B=1$. So, for simplicity, here we deal only with this particular case. Moreover, since the case $\delta_{c}=\delta_{f}$ can be treated similarly, we assume also that $\delta_{c} \neq \delta_{f}$. From the discussion above, we get the candidate pair $\left(u^{*}, X^{*}\right)$ :

$$
\left\{\begin{array}{l}
u_{t}^{*}=\int_{0}^{t} \mathcal{U}_{V}^{*}(t-s) \mathrm{d} V_{s}^{H}-\frac{A}{r} \int_{0}^{t} p_{2}^{*}(t-s) \mathrm{d} W_{s}^{H}, \\
X_{t}^{*}=\int_{0}^{t} \mathcal{X}_{V}^{*}(t-s) \mathrm{d} V_{s}^{H}+\frac{b}{A} \int_{0}^{t} \mathcal{U}_{V}^{*}(t-s) \mathrm{d} W_{s}^{H},
\end{array}\right.
$$

where, for $\Gamma$ and $\Lambda$ defined by (76) and (77), the function $\mathcal{Z}^{*}=\left(\mathcal{X}_{V}^{*}, \mathcal{U}_{V}^{*}\right)^{\prime}$ is the solution of

$$
\dot{\mathcal{Z}}^{*}=-\Lambda^{\prime} \mathcal{Z}^{*}-r^{-1} \mathcal{B B}^{\prime} \Gamma \lambda^{*} ; \quad \mathcal{Z}^{*}(0)=(1,0)^{\prime}
$$

and $p_{2}^{*}$ is the second component of the vector $p^{*}=\Gamma\left(\mathcal{Z}^{*}+\lambda^{*}\right)$ where $\Gamma \lambda^{*}$ corresponds through $(82)$ to $\Gamma \widetilde{\lambda}^{*}$ given by (78)-(79). Actually, applying (82) to (78)-(79), we get that $\Gamma \lambda^{*}$ is given by:

$$
\Gamma \lambda^{*}(t)=\frac{\bar{\rho} \bar{\gamma}}{\delta_{f}-\delta_{c}}\left(\begin{array}{c}
\left(\delta_{f}^{2}-a^{2}\right) \varphi_{c}(t)-\left(\delta_{c}^{2}-a^{2}\right) \varphi_{f}(t) \\
b\left[\left(\delta_{f}-a\right) \varphi_{c}(t)-\left(\delta_{c}-a\right) \varphi_{f}(t)\right]
\end{array}\right)
$$

where

$$
\varphi_{f}(t)=-\frac{\delta_{f}^{\frac{1}{2}-H}}{\Gamma\left(\frac{3}{2}-H\right)} \int_{t}^{\infty} \mathrm{e}^{\delta_{f}(t-s)} \mathrm{d} s^{\frac{1}{2}-H} ; \quad \varphi_{c}(t)=-\frac{\delta_{c}^{\frac{1}{2}-H}}{\Gamma\left(\frac{3}{2}-H\right)} \int_{t}^{\infty} \mathrm{e}^{\delta_{c}(t-s)} \mathrm{d} s^{\frac{1}{2}-H} .
$$

At first, we prove that the cost $J_{\infty}\left(u^{*}\right)$ corresponding to the pair $\left(u^{*}, X^{*}\right)$ defined by (88)-(91) is the optimal cost $J_{\infty}(\bar{u})$ given by $(45)$, i.e.

$$
\lim _{T \rightarrow+\infty} \frac{1}{T} \int_{0}^{T}\left[q X_{t}^{* 2}+r u_{t}^{* 2}\right] \mathrm{d} t=J_{\infty}(\bar{u}) \quad \text { a.s. }
$$

Since by construction of the pair $\left(u^{*}, X^{*}\right)$ we have already guaranteed that

$$
\lim _{t \rightarrow+\infty} \mathbb{E}\left[q X_{t}^{* 2}+r u_{t}^{* 2}\right]=J_{\infty}(\bar{u})
$$

it is sufficient to show that

$$
\lim _{T \rightarrow+\infty} \frac{1}{T} \int_{0}^{T}\left[X_{t}^{* 2}-\mathbb{E} X_{t}^{* 2} \mathrm{~d} t\right]=0 \quad \text { a.s. } ; \quad \lim _{T \rightarrow+\infty} \frac{1}{T} \int_{0}^{T}\left[u_{t}^{* 2}-\mathbb{E} u_{t}^{* 2}\right] \mathrm{d} t=0 \quad \text { a.s. }
$$

To estimate the covariances $\mathbb{E} X_{t}^{*} X_{t+\tau}^{*}$ and $\mathbb{E} u_{t}^{*} u_{t+\tau}^{*}$, we shall take benefit of the following extension of the equality (66) for a stochastic integral $S_{t}=\int_{0}^{t} g(t-s) \mathrm{d} B_{s}^{H}$ : for any $\tau \geq 0$

$$
\lim _{t \rightarrow+\infty} \mathbb{E} S_{t} S_{t+\tau}=\frac{2 H \Gamma\left(\frac{3}{2}-H\right)}{\Gamma\left(H+\frac{1}{2}\right) \Gamma(2-2 H)} \int_{0}^{+\infty} \widetilde{g}(s) \widetilde{g}(s+\tau) \mathrm{d} s,
$$

where $\widetilde{g}$ is defined by (67). Due to $(73)-(74)$ and (78)-(79), it can be seen that there exists a constant ${ }^{2} C>0$ such that for all $s \geq 0$

$$
\left|\widetilde{\mathcal{Z}}^{*}(s)\right| \leq C\left(1 \wedge s^{H-\frac{3}{2}}\right) ; \quad\left|\widetilde{p}^{*}(s)\right| \leq C\left(1 \wedge s^{H-\frac{3}{2}}\right) .
$$

\footnotetext{
${ }^{2}$ We use here and throughout the proof $C$ to denote an unspecified positive constant, not always the same.
} 
So the $\widetilde{g}$ 's corresponding to the $g$ 's involved in the representation (88) of the pair $\left(u^{*}, X^{*}\right)$ in terms of $V^{H}$ and $W^{H}$ satisfy also such an inequality. Hence, from (94), we get that for all $t \geq 0$ and $\tau \geq 0$

$$
\left|\mathbb{E} X_{t}^{*} X_{t+\tau}^{*}\right| \leq C\left(1 \wedge \tau^{2 H-2}\right) ; \quad\left|\mathbb{E} u_{t}^{*} u_{t+\tau}^{*}\right| \leq C\left(1 \wedge \tau^{2 H-2}\right)
$$

Consequently, we can apply assertion (ii) in Proposition 5.1 of the Appendix to the continuous and centered Gaussian processes $X^{*}$ and $u^{*}$ with $\beta=2-2 H>0$ and $k=1$. It gives that the limits in (93) hold, which implies also the validity of (92).

Now we turn to the representation (83)-(87) of the optimal control $u^{*}$. Taking into account the form (41) of $\bar{u}$ in terms of the optimal filter $\pi(\bar{X})$ in the first solution of the problem, it is natural to guess that here a similar decomposition may hold for $u^{*}$ in terms of an asymptotically optimal filter for $X^{*}$. In Remark $4.2(\mathrm{c})$, we have already seen that the process $\pi^{*}=\hat{\pi}+A \bar{\gamma} v_{t}^{f}$, given by (84), (85) and (87), is a candidate to be such a filter. Since it plays a key role in the definition of the terms $\hat{\pi}$ and $v_{t}^{f}$ appearing in the expression of $\pi^{*}$, it seems reasonable to look for a complete representation of $u^{*}$ in terms of the process $Y^{*}-\int_{0}^{*} A \hat{\pi} \mathrm{d} s$. So, as a first step, we look for $u^{*}$ in the form

$$
u_{t}^{*}=\int_{0}^{t} g^{*}(t-s)\left[\mathrm{d} Y_{s}^{*}-A \hat{\pi}_{s} \mathrm{~d} s\right]
$$

Then it can be checked that equation (85) and the first equation in (88) imply that

$$
\hat{\pi}_{t}=\int_{0}^{t} \Psi^{*}(t-s)\left[\mathrm{d} Y_{s}^{*}-A \hat{\pi}_{s} \mathrm{~d} s\right]
$$

with a function $\Psi^{*}$ satisfying

$$
\dot{\Psi}^{*}=a \Psi^{*}+b g^{*} ; \quad \Psi^{*}(0)=A \bar{\gamma}
$$

where $g^{*}$ is given by

$$
g^{*}(t)=A \bar{\gamma} \mathcal{U}_{V}^{*}-\frac{A}{r} p_{2}^{*}(t)
$$

Since $p_{2}^{*}$ is the second component of the vector $p^{*}=\Gamma\left(\mathcal{Z}^{*}+\lambda^{*}\right)$ where $\Gamma$ is given by $(76)$ and $\mathcal{Z}^{*}=\left(\mathcal{X}_{V}^{*}, \mathcal{U}_{V}^{*}\right)^{\prime}$, we get that

$$
g^{*}(t)=\widehat{g}(t)-\frac{A}{r}\left(\Gamma \lambda^{*}\right)_{2}(t),
$$

where $\left(\Gamma \lambda^{*}\right)_{2}$ is the second component of the vector $\Gamma \lambda^{*}$ given by (90) and

$$
\widehat{g}(t)=-\frac{a+\delta_{c}}{A}\left[\frac{a+\delta_{f}}{b} \mathcal{X}_{V}^{*}(t)+\mathcal{U}_{V}^{*}(t)\right] .
$$

Now, from equation (89), it comes that

$$
\dot{\widehat{g}}=-\delta_{c} \widehat{g}+\frac{A}{r}\left(a+\delta_{c}\right)\left(\Gamma \lambda^{*}\right)_{2} ; \quad \widehat{g}(0)=-\frac{a+\delta_{c}}{b} \cdot \frac{a+\delta_{f}}{A},
$$

which can be rewritten as

$$
\dot{\hat{g}}=a \widehat{g}-\left(a+\delta_{c}\right) g^{*} ; \quad \widehat{g}(0)=-\frac{a+\delta_{c}}{b} \cdot \frac{a+\delta_{f}}{A} .
$$

Since $\frac{a+\delta_{c}}{b}=\frac{b}{r} \bar{\rho}$ and $\frac{a+\delta_{f}}{A}=A \bar{\gamma}$, a comparison of equations (97) and (99) gives that $\widehat{g}=-\frac{b}{r} \bar{\rho} \Psi^{*}$ and hence, due to (98), we have

$$
g^{*}(t)=-\frac{b}{r} \bar{\rho} \Psi^{*}-\frac{A}{r}\left(\Gamma \lambda^{*}\right)_{2}(t)
$$


Inserting this equality into (95) and taking into account (96), it follows that

$$
u_{t}^{*}=-\frac{b}{r} \bar{\rho} \hat{\pi}_{t}-\frac{A}{r} \int_{0}^{t}\left(\Gamma \lambda^{*}\right)_{2}(t-s)\left[\mathrm{d} Y_{s}^{*}-A \hat{\pi}_{s} \mathrm{~d} s\right] .
$$

But from (90) we have

$$
\left(\Gamma \lambda^{*}\right)_{2}(t)=\frac{b \bar{\rho} \bar{\gamma}}{\delta_{f}-\delta_{c}}\left\{\left(\delta_{f}-a\right) \varphi_{c}(t)-\left(\delta_{c}-a\right) \varphi_{f}(t)\right\},
$$

where $\varphi_{f}$ and $\varphi_{c}$ are given by (91). It can be checked that actually the processes

$$
v_{c}(t)=\int_{0}^{t} \frac{\delta_{c}^{\frac{1}{2}-H}}{\Gamma\left(\frac{3}{2}-H\right)} \varphi_{c}(t-\tau)\left[\mathrm{d} Y_{\tau}-A \hat{\pi}_{\tau} \mathrm{d} \tau\right],
$$

and

$$
v_{f}(t)=\int_{0}^{t} \frac{\delta_{f}^{\frac{1}{2}-H}}{\Gamma\left(\frac{3}{2}-H\right)} \varphi_{f}(t-\tau)\left[\mathrm{d} Y_{\tau}-A \hat{\pi}_{\tau} \mathrm{d} \tau\right],
$$

are nothing but the solutions of equations (86) and (87) respectively. Therefore, we can write

$$
u_{t}^{*}=-\frac{b}{r} \bar{\rho}\left\{\hat{\pi}_{t}+\frac{A \bar{\gamma}}{\delta_{c}-\delta_{f}}\left[\left(\delta_{c}-a\right) v_{t}^{f}-\left(\delta_{f}-a\right) v_{t}^{c}\right]\right\} .
$$

Finally, using (84), it is readily seen that $u_{t}^{*}$ can be rewritten as in (83).

\section{ApPEndiX - AuXiLiary Results}

\subsection{Some sufficient condition on moments for ergodic type properties of processes}

The following sufficient condition for ergodic type properties is the key of several steps in our developments. It has already been used in the proof of Theorem 4.1 and it will be again repeatedly used below. Notice that throughout the statements and the proofs, below we use $C$ to denote an unspecified positive constant, not always the same.

Proposition 5.1. Let $\xi=\left(\xi_{t}, t \geq 0\right)$ be a centered continuous process. Suppose that there exists some constants $C>0$ and $\beta>0$ such that the condition

$$
\left|\mathbb{E} \xi_{t} \xi_{t+\tau}\right| \leq C\left(1 \wedge \tau^{-\beta}\right),
$$

holds for all $t \geq 0$ and $\tau \geq 0$. Then the process $\left(\xi_{t}, t \geq 0\right)$ possesses the following ergodic type properties:

(i)

$$
\lim _{T \rightarrow+\infty} \frac{1}{T} \int_{0}^{T} \xi_{t} \mathrm{~d} t=0 \quad \text { a.s. }
$$

(ii) if moreover $\xi$ is Gaussian, then for any integer $k \geq 1$,

$$
\lim _{T \rightarrow+\infty} \frac{1}{T} \int_{0}^{T}\left[\xi_{t}^{2 k}-\mathbb{E} \xi_{t}^{2 k}\right] \mathrm{d} t=0 \quad \text { a.s. }
$$

Proof. For $0<\beta<1$, assertion $(i)$ is actually an immediate consequence of the last statement written on page 95 in [2] since if the above condition (100) is satisfied then the condition $0 \leq 2 \alpha<\beta<1$ numbered (5.5.3) 
therein is obviously fulfilled with $\alpha=0$. For $\beta \geq 1$, it is clear that the condition (100) is stronger than for $0<\beta<1$ and so the conclusion remains still valid.

Now, if the centered process $\xi$ is Gaussian, for any $k \geq 1$, we have

$$
\left|\mathbb{E}\left[\xi_{t}^{2 k}-\mathbb{E} \xi_{t}^{2 k}\right]\left[\xi_{t+\tau}^{2 k}-\mathbb{E} \xi_{t+\tau}^{2 k}\right]\right| \leq C\left[\mathbb{E} \xi_{t} \xi_{t+\tau}\right]^{2 k},
$$

with some positive constant C, and hence also, if $\xi$ satisfies (100), then

$$
\left|\mathbb{E}\left[\xi_{t}^{2 k}-\mathbb{E} \xi_{t}^{2 k}\right]\left[\xi_{t+\tau}^{2 k}-\mathbb{E} \xi_{t+\tau}^{2 k}\right]\right| \leq C\left(1 \wedge \tau^{-2 k \beta}\right)
$$

Consequently, assertion ( $i$ i ) follows by a simple application of the first statement in the Proposition to the centered process $\left(\xi_{t}^{2 k}-\mathbb{E} \xi_{t}^{2 k}, t \geq 0\right)$ with $\beta$ changed into $2 k \beta$.

\subsection{About some technical results used in the proof of Theorem 3.1}

Here we are concerned with a system with constant coefficients. Again, without any loss of generality and for simplicity, we assume that $\sigma=B=1$ and $x=0$. Moreover, since the case $\delta_{c}=\delta_{f}$ can be treated similarly, we assume also that $\delta_{c} \neq \delta_{f}$. For the pair $(X, Y)$ solution of the system (1), we introduce the filtering error process $\Delta$ as

$$
\Delta_{t}=X_{t}-\pi_{t}(X), \quad t \geq 0 .
$$

Recall that this process, which is centered, does not depend on the specific control $u$ involved in the system. Hence, to analyze the second order structure and the asymptotic behavior of $\Delta$, we may deal only with the case $u \equiv 0$. Notice that, from the asymptotical point of view, from [8], we know already that for $t$ tending to infinity the variance function $\Gamma_{f}^{11}(t, t)=\mathbb{E} \Delta_{t}^{2}$ behaves as follows:

$$
\lim _{t \rightarrow+\infty} \mathbb{E} \Delta_{t}^{2}=\gamma_{\infty}(H)
$$

where $\gamma_{\infty}(H)$ is the constant given by (47). But, in order to derive ergodic type properties of $\Delta$ on the basis of Proposition 5.1, we need a much deeper analysis of the behavior of the covariance function $\mathbb{E} \Delta_{t} \Delta_{s}$. Preparing for that, we give a key representation of this covariance function in terms of covariances of some other processes. Hereafter, the $2 \times 2$ matrices $A_{H}, B_{H}, J$ and the vector $b_{H}$ in $\mathbb{R}^{2}$ are defined by:

$$
A_{H}(s)=\left(\begin{array}{cc}
1 & s^{1-2 H} \\
s^{2 H-1} & 1
\end{array}\right) ; \quad B_{H}(s)=\left(\begin{array}{cc}
s^{1-2 H} & 1 \\
1 & s^{2 H-1}
\end{array}\right)
$$

and

$$
J=\left(\begin{array}{ll}
0 & 1 \\
1 & 0
\end{array}\right) ; \quad b_{H}(s)=\left(\begin{array}{c}
1 \\
s^{2 H-1}
\end{array}\right) .
$$

Following [8], we introduce the auxiliary process $\zeta=\left(\zeta_{t}, t \geq 0\right)$ as the solution of the equation

$$
\zeta_{t}=\frac{a}{2} \int_{0}^{t} A_{H}(s) \zeta_{s} \mathrm{~d} s+\int_{0}^{t} b_{H}(s) \mathrm{d} V_{s}^{*}
$$

in which $V^{*}$ denotes the fundamental martingale associated to $V^{H}$ through (15). In terms of $\zeta$, the process $Q$ defined by (19) can be represented as

$$
Q(t)=\frac{A \lambda_{H}}{2(2-2 H)} b_{H}^{\prime}(t) J \zeta_{t},
$$


and, for all fixed $t \geq 0$, we define another auxiliary process $X^{t}$ on $[0, t]$ as the solution of the equation

$$
X_{s}^{t}=\frac{a}{2} \int_{0}^{t} K_{H}(t, u) b_{H}^{\prime}(u) J \zeta_{u} \mathrm{~d} u+\int_{0}^{s} K_{H}(t, u) \mathrm{d} V_{u}^{*} ; \quad 0 \leq s \leq t .
$$

From [8], we know that the covariance function $\gamma_{\zeta \zeta}$ defined by

$$
\gamma_{\zeta \zeta}(s)=\mathbb{E}\left(\zeta_{s}-\pi_{s}(\zeta)\right)\left(\zeta_{s}-\pi_{s}(\zeta)\right)^{\prime}, s \geq 0,
$$

is the solution of the Riccati equation

$$
\begin{aligned}
\gamma_{\zeta \zeta}(s)= & \frac{a}{2} \int_{0}^{s}\left[A_{H}(u) \gamma_{\zeta \zeta}(u)+\gamma_{\zeta \zeta}(u) A_{H}^{\prime}(u)\right] \mathrm{d} u \\
& +\frac{2-2 H}{\lambda_{H}} \int_{0}^{s} B_{H}(u) \mathrm{d} u-\frac{A^{2} \lambda_{H}}{4(2-2 H)} \int_{0}^{s} \gamma_{\zeta \zeta}(u) B_{1-H}(u) \gamma_{\zeta \zeta}(u) \mathrm{d} u .
\end{aligned}
$$

Reworking the developments in [8], one can show that the covariances

$$
\gamma_{X \zeta}(t, s)=\mathbb{E}\left(X_{s \wedge t}^{t}-\pi_{s \wedge t}\left(X^{t}\right)\right)\left(\zeta_{s}-\pi_{s}(\zeta)\right), s \geq 0, t \geq 0,
$$

satisfy for all $s \geq 0$ and $t \geq 0$ the equation

$$
\begin{aligned}
\gamma_{X \zeta}(t, s)= & \int_{0}^{t}\left\{\frac{a}{2} A_{H}(u)-\frac{A^{2} \lambda_{H}}{4(2-2 H)} \gamma_{\zeta \zeta}(u) B_{1-H}(u)\right\} \gamma_{X \zeta}(t, u) \mathrm{d} u \\
& +\int_{0}^{s \wedge t} K_{H}(t, u)\left\{\frac{a}{2} \gamma_{\zeta \zeta}(u)+\frac{2-2 H}{\lambda_{H}} J\right\} b_{1-H}(u) \mathrm{d} u .
\end{aligned}
$$

Finally, in terms of the previous covariances, one can derive the following representation of the covariance $\mathbb{E} \Delta_{t} \Delta_{s}$ : for all $0 \leq s \leq t$

$$
\begin{aligned}
\mathbb{E} \Delta_{t} \Delta_{s}= & \int_{0}^{s} K_{H}(s, r) K_{H}(t, r) \mathrm{d} w_{r}^{H}+\frac{a}{2} \int_{0}^{s} K_{H}(s, r) b_{1-H}^{\prime}(r) \gamma_{X \zeta}(t, r) \mathrm{d} r \\
& +\int_{0}^{t}\left\{\frac{a}{2} K_{H}(t, r) b_{1-H}^{\prime}(r)-\frac{A^{2} \lambda_{H}}{4(2-2 H)} \gamma_{X \zeta}^{\prime}(t, r) B_{1-H}(r)\right\} \gamma_{X \zeta}(s, r) \mathrm{d} r .
\end{aligned}
$$

Now, on the basis of this representation and Proposition 5.1, we are able to prove the following statement which provides ergodic type properties of the process $\Delta$.

Lemma 5.2. Let $\Delta$ be the process defined by (101) for the solution pair $(X, Y)$ of the system (1). Then the following assertions hold:

(i) there exists a constant $C>0$ such that for all $t \geq 0$ and $\tau \geq 0$

$$
\left|\mathbb{E} \Delta_{t} \Delta_{t+\tau}\right| \leq C\left(1 \wedge \tau^{2 H-2}\right),
$$

(ii) the process $\Delta$ possesses the ergodic type property

$$
\lim _{T \rightarrow+\infty} \frac{1}{T} \int_{0}^{T} \Delta_{t}^{2} \mathrm{~d} t=\gamma_{\infty}(H) \quad \text { a.s. }
$$

where $\gamma_{\infty}(H)$ is given by (47), 
(iii) if $v=\left(v_{t}, t \geq 0\right)$ is a $\left(\mathcal{Y}_{t}\right)$-adapted continuous process such that almost surely $\limsup _{T \rightarrow+\infty} T^{-1} \int_{0}^{T} v_{t}^{2} \mathrm{~d} t<+\infty$, then

$$
\lim _{T \rightarrow+\infty} \frac{1}{T} \int_{0}^{T} v_{t} \Delta_{t} \mathrm{~d} t=0 \quad \text { a.s. }
$$

Proof. Concerning assertion $(i)$, since the complete proof is quite long and very technical, here we just give some hints about the main steps. ${ }^{3}$ At first, in the representation (106), explicite expressions of the involved solutions of equations (104)-(105) can be obtained. Then, from these expressions, refining the methods used in [8], precise limiting properties of the various terms inside the integrals in the right hand side of (106) can be analyzed. Finally, it can be shown that for any fixed $\tau \geq 0$,

$$
\lim _{t \rightarrow+\infty} \mathbb{E} \Delta_{t} \Delta_{t+\tau}=\gamma_{\Delta}(\tau),
$$

where the limit $\gamma_{\Delta}(\tau)$ is finite and explicit, with of course in particular $\gamma_{\Delta}(0)=\gamma_{\infty}(H)$ given by $(47)$. Moreover, a complementary study gives that

$$
\lim _{\tau \rightarrow+\infty} \tau^{2-2 H} \gamma_{\Delta}(\tau)=\gamma_{\Delta}
$$

where again the limit $\gamma_{\Delta}$ is finite and can be written explicitly. Hence, it is readily seen that the assertion $(i)$ holds.

Now we prove assertion (ii). Since $\Delta$ is a centered Gaussian process and, due to (102), we have

$$
\lim _{T \rightarrow+\infty} \frac{1}{T} \int_{0}^{T}\left[\mathbb{E} \Delta_{t}^{2}\right] \mathrm{d} t=\lim _{t \rightarrow+\infty} \mathbb{E} \Delta_{t}^{2}=\gamma_{\infty}(H),
$$

(108) follows by a simple application of Proposition 5.1 (ii) with $\beta=2-2 H$ and $k=1$.

Finally we turn to assertion (iii). At first, we prove (109) for a bounded process $v$. Since $v$ is $\left(\mathcal{Y}_{t}\right)$-adapted and $\Delta$ is centered, $\mathbb{E} v_{t} \Delta_{t}=0$ for all $t \geq 0$, i.e., the process $v \Delta$ is centered. Since $\Delta$ is Gaussian, for all $t \geq 0$ and $\tau \geq 0$, the conditional covariance $\mathbb{E}\left[\Delta_{t} \Delta_{t+\tau} / \mathcal{Y}_{t+\tau}\right]$ is deterministic and hence

$$
\mathbb{E} v_{t} v_{t+\tau} \Delta_{t} \Delta_{t+\tau}=\mathbb{E}\left[\mathbb{E}\left[v_{t} v_{t+\tau} \Delta_{t} \Delta_{t+\tau} / \mathcal{Y}_{t+\tau}\right]\right]=\mathbb{E} v_{t} v_{t+\tau} \mathbb{E} \Delta_{t} \Delta_{t+\tau}
$$

So, if $\sup _{t \geq 0}\left|v_{t}\right| \leq C<\infty$ a.s., then for all $t \geq 0$ and $\tau \geq 0$

$$
\left|\mathbb{E} v_{t} v_{t+\tau} \Delta_{t} \Delta_{t+\tau}\right|=\left|\mathbb{E} v_{t} v_{t+\tau}\right|\left|\mathbb{E} \Delta_{t} \Delta_{t+\tau}\right| \leq C\left(1 \wedge \tau^{2 H-2}\right) .
$$

Consequently, by application of Proposition 5.1 (i) to $v \Delta$ with $\beta=2-2 H$, we get that (109) holds. Now, we consider the case of a possibly unbounded $v$. For any fixed positive constant $K$, we can write

$$
\frac{1}{T} \int_{0}^{T} v_{t} \Delta_{t} \mathrm{~d} t=I_{1}^{K}(T)+I_{2}^{K}(T)
$$

where

$$
I_{1}^{K}(T)=\frac{1}{T} \int_{0}^{T} I\left(\left|v_{t}\right| \leq K\right) v_{t} \Delta_{t} \mathrm{~d} t ; \quad I_{2}^{K}(T)=\frac{1}{T} \int_{0}^{T} v_{t} I\left(\left|v_{t}\right|>K\right) \Delta_{t} \mathrm{~d} t .
$$

Since the process $\left(I\left(\left|v_{t}\right| \leq K\right) v_{t}, t \geq 0\right)$ is bounded, by the first step above, we know that $I_{1}^{K}(T)$ tends a.s. to zero as $T$ goes to infinity and so we obtain that for all $K>0$

$$
\limsup _{T \rightarrow+\infty}\left|\frac{1}{T} \int_{0}^{T} v_{t} \Delta_{t} \mathrm{~d} t\right| \leq \limsup _{T \rightarrow+\infty}\left|I_{2}^{K}(T)\right| \quad \text { a.s. }
$$

\footnotetext{
${ }^{3}$ Further details can be obtained upon request to the author for correspondence.
} 
Hence, to prove that (109) is still valid, it suffices to show that

$$
\lim _{K \rightarrow+\infty} \limsup _{T \rightarrow+\infty}\left|I_{2}^{K}(T)\right|=0 \quad \text { a.s. }
$$

But, using the Cauchy-Schwarz inequality, we see that

$$
\left|I_{2}^{K}(T)\right| \leq \sqrt{\frac{1}{T} \int_{0}^{T} v_{t}^{2} \mathrm{~d} t} \sqrt[4]{\frac{1}{T} \int_{0}^{T} I\left(\left|v_{t}\right|>K\right) \mathrm{d} t} \sqrt[4]{\frac{1}{T} \int_{0}^{T} \Delta_{t}^{4} \mathrm{~d} t}
$$

In the second factor of the right hand side, we have

$$
\frac{1}{T} \int_{0}^{T} I\left(\left|v_{t}\right|>K\right) \mathrm{d} t \leq \frac{1}{K T} \int_{0}^{T}\left|v_{t}\right| \mathrm{d} t \leq \frac{1}{K} \sqrt{\frac{1}{T} \int_{0}^{T} v_{t}^{2} \mathrm{~d} t}
$$

and so, we get

$$
\left|I_{2}^{K}(T)\right| \leq \frac{1}{\sqrt[4]{K}}\left[\frac{1}{T} \int_{0}^{T} v_{t}^{2} \mathrm{~d} t\right]^{\frac{5}{8}}\left[\frac{1}{T} \int_{0}^{T} \Delta_{t}^{4} \mathrm{~d} t\right]^{\frac{1}{4}} .
$$

Since $\Delta$ is a centered Gaussian process, we have $\mathbb{E} \Delta_{t}^{4}=3\left(\mathbb{E} \Delta_{t}^{2}\right)^{2}$ and hence, due to (102), it follows that

$$
\lim _{T \rightarrow+\infty} \frac{1}{T} \int_{0}^{T}\left[\mathbb{E} \Delta_{t}^{4}\right] \mathrm{d} t=\lim _{t \rightarrow+\infty} \mathbb{E} \Delta_{t}^{4}=3 \gamma_{\infty}^{2}(H) .
$$

Then, applying Proposition 5.1 (ii) with $\beta=2-2 H$ and $k=2$ to $\Delta$, it turns that

$$
\lim _{T \rightarrow+\infty} \frac{1}{T} \int_{0}^{T} \Delta_{t}^{4} \mathrm{~d} t=3 \gamma_{\infty}^{2}(H) \quad \text { a.s. }
$$

Finally, thanks to the assumption $\lim \sup _{T \rightarrow+\infty} T^{-1} \int_{0}^{T} v_{t}^{2} \mathrm{~d} t<+\infty$ a.s., the inequality (111) shows that (110) is valid and hence also (109) holds for $v$.

Lemma 5.3. Let the kernel $\Gamma_{f}^{12}(t, s)$ be the $(1,2)$-entry of $\Gamma_{f}(t, s)$ defined by (21) with $\mathcal{A}_{f}$ and $\mathcal{C}$ given by (38). Let $\bar{z}=\left(\bar{z}_{t}, t \geq 0\right)$ be the process driven by the equation

$$
\bar{z}_{t}=-\delta_{c} \int_{0}^{t} \bar{z}_{s} \mathrm{~d} s+\int_{0}^{t} \Gamma_{f}^{12}(t, s) \mathrm{d} \nu_{s} .
$$

Then the following assertions hold:

(i) for all $\tau \geq 0$

where

$$
\lim _{t \rightarrow+\infty} \sqrt{\frac{2-2 H}{\lambda_{H}}} t^{\frac{1}{2}-H} \Gamma_{f}^{12}(t+\tau, t)=\Gamma_{\star}(\tau),
$$

$$
\Gamma_{\star}(\tau)=A \sqrt{\Gamma(2 H+1) \sin \pi H}\left\{\frac{a \bar{\gamma}}{\delta_{f}^{H+\frac{1}{2}}}+\frac{1}{\Gamma\left(H+\frac{1}{2}\right)} \int_{0}^{+\infty} \mathrm{e}^{-\delta_{f} s}(\tau+s)^{H-\frac{1}{2}} \mathrm{~d} s\right\},
$$

(ii) there exists a constant $C>0$ such that for all $t \geq 0$ and $\tau \geq 0$

$$
\left|\mathbb{E} \bar{z}_{t} \bar{z}_{t+\tau}\right| \leq C\left(1 \wedge \tau^{2 H-2}\right),
$$


(iii) the process $\bar{z}$ possesses the ergodic type property

$$
\begin{aligned}
\lim _{T \rightarrow+\infty} \frac{1}{T} \int_{0}^{T} \bar{z}_{t}^{2} \mathrm{~d} t= & \frac{A^{2} \Gamma(2 H+1)}{2\left(\delta_{f}^{2}-\delta_{c}^{2}\right)}\left[\frac{1}{\delta_{c}^{2 H}}-\frac{1}{\delta_{f}^{2 H}}\right] \\
& +A^{2} \Gamma(2 H+1) \sin \pi H\left\{\frac{\delta_{f}^{1-2 H}}{\left(\delta_{c}+\delta_{f}\right)^{2}}\left[\frac{\bar{\gamma}^{2}\left(\delta_{c}+a\right)^{2}}{2 \delta_{c}}+\frac{\left.2 \bar{\gamma}_{c}+a\right)}{\delta_{c}-\delta_{f}}-\frac{1}{2 \delta_{f}}\right]\right. \\
& \left.+\frac{\delta_{f}^{\frac{1}{2}-H} \delta_{c}^{-\frac{1}{2}-H}}{\delta_{f}^{2}-\delta_{c}^{2}} \bar{\gamma}\left(\delta_{c}+a\right)\right\} \text { a.s. }
\end{aligned}
$$

Proof. At first we deal with assertion $(i)$. Here again, the complete proof is rather long and very technical and so we just give some hints. ${ }^{4}$ The first step is to derive an appropriate representation of the kernel $\Gamma_{f}^{12}(t, s)$. From [9], we know that if $\widetilde{X}^{t}$ denotes the auxiliary process defined on $[0, t]$ by

$$
\tilde{X}_{s}^{t}=a \int_{0}^{s} X_{r} \mathrm{~d} r+\int_{0}^{s} K_{H}(t, r) \mathrm{d} V_{r}^{*} ; \quad 0 \leq s \leq t,
$$

in which $V^{*}$ stands for the fundamental martingale associated to the $\mathrm{fBm} V^{H}$ through (15), then

$$
\Gamma_{f}^{12}(t, s)=\mathbb{E}\left(\widetilde{X}_{s}^{t}-\pi_{s}\left(\widetilde{X}^{t}\right)\right)\left(Q_{s}-\pi_{s}(Q)\right) ; \quad 0 \leq s \leq t,
$$

where $Q$ is the process defined by (19). Now, due to the representation (103) of $Q$, for $0 \leq s \leq t$, the kernel $\Gamma_{f}^{12}(t, s)$ is given by

$$
\Gamma_{f}^{12}(t, s)=\frac{A \lambda_{H}}{2(2-2 H)} b_{H}^{\prime}(s) J \Gamma_{X \zeta}(t, s),
$$

where the vectors $\Gamma_{X \zeta}(t, s)$ in $\mathbb{R}^{2}$ are the covariances

$$
\Gamma_{X \zeta}(t, s)=\mathbb{E}\left(\widetilde{X}_{s}^{t}-\pi_{s}\left(\widetilde{X}^{t}\right)\right)\left(\zeta_{s}-\pi_{s}(\zeta)\right) ; \quad 0 \leq s \leq t
$$

It can be shown that these covariances satisfy the equation

$$
\begin{aligned}
\Gamma_{X \zeta}(t, s) & =a \int_{0}^{s} \Gamma_{X \zeta}(r, r) \mathrm{d} r+\frac{2-2 H}{\lambda_{H}} \int_{0}^{s} K_{H}(t, r) J b_{1-H}(r) \mathrm{d} r \\
& +\int_{0}^{s}\left\{\frac{a}{2} A_{H}(r)-\frac{A^{2} \lambda_{H}}{4(2-2 H)} \gamma_{\zeta \zeta}(r) B_{1-H}(r)\right\} \Gamma_{X \zeta}(t, r) \mathrm{d} r,
\end{aligned}
$$

where $\gamma_{\zeta \zeta}(r)$ is the solution of equation (104). An explicit expression can be obtained for $\Gamma_{X \zeta}(t, s)$ and then the asymptotic behavior of $\Gamma_{f}^{12}(t+\tau, t)$ can be analyzed from (114) by means of refinements of the methods used in $[8]$.

Now, we prove assertion $(i i)$. Due to equation (112), we get that $\bar{z}$ can be represented as

$$
\bar{z}_{t}=\int_{0}^{t} G(t, s) \mathrm{d} \nu_{s}
$$

where the kernel $G(t, s)$ is given by

$$
G(t, s)=\Gamma_{f}^{12}(t, s)-\delta_{c} \int_{s}^{t} \mathrm{e}^{-\delta_{c}(t-r)} \Gamma_{f}^{12}(r, s) \mathrm{d} r .
$$

\footnotetext{
${ }^{4}$ Further details can be obtained upon request to the author for correspondence.
} 
For all $t \geq 0$ and $\tau \geq 0$, we can write

$$
G(t+\tau, t)=\Gamma_{f}^{12}(t+\tau, t)-\delta_{c} \int_{0}^{\tau} \mathrm{e}^{-\delta_{c}(\tau-u)} \Gamma_{f}^{12}(t+u, t) \mathrm{d} u .
$$

Hence, thanks to assertion (i), after checking that the theorem of dominated convergence can be applied, it comes that

$$
\lim _{t \rightarrow+\infty} \sqrt{\frac{2-2 H}{\lambda_{H}}} t^{\frac{1}{2}-H} G(t+\tau, t)=G_{\star}(\tau)
$$

where

$$
G_{\star}(\tau)=\Gamma_{\star}(\tau)-\delta_{c} \int_{0}^{\tau} \mathrm{e}^{-\delta_{c}(\tau-u)} \Gamma_{\star}(u) \mathrm{d} u,
$$

or, equivalently,

$$
G_{\star}(\tau)=\mathrm{e}^{-\delta_{c} \tau} \Gamma_{\star}(0)+\int_{0}^{\tau} \mathrm{e}^{-\delta_{c}(\tau-u)} \dot{\Gamma}_{\star}(u) \mathrm{d} u .
$$

Now, concerning the covariance function of the process $\bar{z}$, from equation (115), we have

$$
\mathbb{E} \bar{z}_{t} \bar{z}_{t+\tau}=\int_{0}^{t} G(t, s) G(t+\tau, s) \mathrm{d}\langle\nu\rangle_{s} ; \quad \tau \geq 0 .
$$

Consequently, since the variance function $\langle\nu\rangle$ of the process $\nu$ is nothing but the function $w^{H}$ given by (14) and moreover again the theorem of dominated convergence is applicable, it follows from (116) that

$$
\lim _{t \rightarrow+\infty} \mathbb{E} \bar{z}_{t} \bar{z}_{t+\tau}=\int_{0}^{+\infty} G_{\star}(r) G_{\star}(r+\tau) \mathrm{d} r ; \quad \tau \geq 0 .
$$

Actually, it can be seen that there exists a constant $C>0$ such that for all $s \geq 0$

$$
\left|G_{\star}(s)\right| \leq C\left(1 \wedge s^{H-\frac{3}{2}}\right),
$$

and so we get also that for all $t \geq 0$ and $\tau \geq 0$

$$
\left|\mathbb{E} \bar{z}_{t} \bar{z}_{t+\tau}\right| \leq C\left(1 \wedge \tau^{2 H-2}\right),
$$

which means that assertion (ii) is valid.

Finally, we turn to assertion (iii). Here, since the process $\bar{z}$ is Gaussian and centered, due to Proposition 5.1 (ii) and assertion (ii) above, it suffices to show that the limit of $\mathbb{E} \bar{z}_{t}^{2}$ as $t$ goes to infinity is equal to the right hand side of the equality stated in assertion (iii). We already know that this limit is given by

$$
\lim _{t \rightarrow+\infty} \mathbb{E} \bar{z}_{t}^{2}=\int_{0}^{+\infty} G_{\star}^{2}(r) \mathrm{d} r,
$$

where $G_{\star}$ satisfies equation (117) and so it remains to calculate the integral in the right hand side. But, from equations (113) and (117), we can write a differential system for the pair $\left(G_{\star}, \dot{\Gamma}_{\star}\right)$ and then the integral appears as the value of the cost function corresponding to a specific control policy in some infinite time horizon linear quadratic control problem. Finally, this value can be computed by applying a standard method in such a setting. 
Lemma 5.4. Let the function $\gamma_{c}^{12}(s, s)$ be defined by (40) and $\bar{w}=\left(\bar{w}_{t}, t \geq 0\right)$ be the process driven by the equation

Let $\gamma_{\star}$ be the constant

$$
\bar{w}_{t}=-\delta_{c} \int_{0}^{t} \bar{w}_{s} \mathrm{~d} s+\int_{0}^{t} \gamma_{c}^{12}(s, s) \mathrm{d} \nu_{s}
$$

$$
\gamma_{\star}=A \sqrt{\Gamma(2 H+1) \sin \pi H}\left\{\frac{a \bar{\gamma}}{\delta_{f}^{H+\frac{1}{2}}}+\frac{\delta_{c}}{\delta_{f}-\delta_{c}}\left[\frac{1}{\delta_{c}^{H+\frac{1}{2}}}-\frac{1}{\delta_{f}^{H+\frac{1}{2}}}\right]\right\} .
$$

Then the following assertions hold:

(i)

$$
\lim _{t \rightarrow+\infty} \sqrt{\frac{2-2 H}{\lambda_{H}}} t^{\frac{1}{2}-H} \gamma_{c}^{12}(t, t)=\gamma_{\star}
$$

(ii)

(iii)

$$
\lim _{t \rightarrow+\infty} \frac{\bar{w}_{t}}{\sqrt{t}}=0 \quad \text { a.s. }
$$

$$
\lim _{T \rightarrow+\infty} \frac{1}{T} \int_{0}^{T} \bar{w}_{t}^{2} \mathrm{~d} t=\frac{\gamma_{\star}^{2}}{2 \delta_{c}} \quad \text { a.s. }
$$

Proof. From the definition (40), we have

$$
\gamma_{c}^{12}(t, t)=\delta_{c} \int_{0}^{+\infty} \mathrm{e}^{-\delta_{c} \tau} \Gamma_{f}^{12}(t+\tau, t) \mathrm{d} \tau
$$

and so, due to assertion $(i)$ in Lemma 5.3,

$$
\lim _{t \rightarrow+\infty} \sqrt{\frac{2-2 H}{\lambda_{H}}} t^{\frac{1}{2}-H} \gamma_{c}^{12}(t, t)=\delta_{c} \int_{0}^{+\infty} \mathrm{e}^{-\delta_{c} \tau} \Gamma_{\star}(\tau) \mathrm{d} \tau,
$$

where $\Gamma_{\star}(\tau)$ is given by (113). Then, by computing the integral in the right hand side, one gets that assertion $(i)$ is valid with the constant $\gamma_{\star}$ given by (119).

Now, to prove assertions $(i i)$ and (iii), we parallel the proof of Proposition 4.1 in [12]. At first, we recall that the variance function $\langle\nu\rangle$ of the process $\nu$ is nothing but the function $w^{H}$ given by (14). Then, from assertion $(i)$, we have also

$$
\lim _{T \rightarrow+\infty} \frac{1}{T} \int_{0}^{T}\left[\gamma_{c}^{12}(t, t)\right]^{2} \mathrm{~d}\langle\nu\rangle_{t}=\gamma_{\star}^{2} ; \quad \lim _{T \rightarrow+\infty} \frac{2 \delta_{c}}{\mathrm{e}^{2 \delta_{c} T}} \int_{0}^{T} \mathrm{e}^{2 \delta_{c} t}\left[\gamma_{c}^{12}(t, t)\right]^{2} \mathrm{~d}\langle\nu\rangle_{t}=\gamma_{\star}^{2} .
$$

Due to equation (118), we can write

$$
\bar{w}_{T}=\mathrm{e}^{-\delta_{c} T} \int_{0}^{T} \mathrm{e}^{\delta_{c} t} \gamma_{c}^{12}(t, t) \mathrm{d} \nu_{t}
$$

Hence, taking into account the second limiting property in (120), assertion (ii) follows directly by application of the law of iterated logarithm for continuous martingales.

To prove assertion $(\mathrm{iii})$, we apply the Itô formula to the process $\left(\bar{w}_{t}^{2}, t \in[0, T]\right)$ to get the representation

$$
\bar{w}_{T}^{2}=-2 \delta_{c} \int_{0}^{T} \bar{w}_{t}^{2} \mathrm{~d} t+2 \int_{0}^{T} \gamma_{c}^{12}(t, t) \bar{w}_{t} \mathrm{~d} \nu_{t}+\int_{0}^{T}\left[\gamma_{c}^{12}(t, t)\right]^{2} \mathrm{~d}\langle\nu\rangle_{t} .
$$


This can be rewritten as

$$
\left[\frac{1}{T} \int_{0}^{T} \bar{w}_{t}^{2} \mathrm{~d} t\right]\left[1-\frac{L_{T}}{\langle L\rangle_{T}} \Psi_{T}\right]=-\frac{\bar{w}_{T}^{2}}{2 \delta_{c} T}+\frac{1}{2 \delta_{c} T} \int_{0}^{T}\left[\gamma_{c}^{12}(t, t)\right]^{2} \mathrm{~d}\langle\nu\rangle_{t},
$$

where

$$
L_{T}=\int_{0}^{T} \gamma_{c}^{12}(t, t) \bar{w}_{t} \mathrm{~d} \nu_{t} ; \quad\langle L\rangle_{T}=\int_{0}^{T}\left[\gamma_{c}^{12}(t, t)\right]^{2} \bar{w}_{t}^{2} \mathrm{~d}\langle\nu\rangle_{t} ; \quad \Psi_{T}=\frac{\langle L\rangle_{T}}{\delta_{c} \int_{0}^{T} \bar{w}_{t}^{2} \mathrm{~d} t} .
$$

Thanks to the first limiting property in (120) and assertion (ii), we see that the right hand side in (121) tends to $\frac{\gamma_{\star}^{2}}{2 \delta_{c}}$ as $T$ goes to $+\infty$. Now, in the left hand side of (121), we observe that due to assertion $(i)$, we have

$$
0<\lim _{T \rightarrow+\infty} \Psi_{T}<\infty \quad \text { a.s. }
$$

Therefore, making use of Lemma 2.6.3 in [14] to discuss the behavior of the second factor within brackets, it is readily seen that for the first one the statement (iii) holds.

\subsection{Last steps in the proof of Theorem 3.1}

Proof of Properties (53)-(55). Here we prove the key properties used to compute the cost corresponding to the control $\bar{u}$ defined by (41) and (43). Recall that we can write $\bar{u}_{t}=-\frac{b}{r} p_{t}$ where $p_{t}=\bar{\rho}\left[\pi_{t}(\bar{X})+\bar{v}_{t}\right]$ with

$$
\bar{v}_{t}=\int_{0}^{t}\left[\gamma_{c}^{12}(t, s)-\Gamma_{f}^{12}(t, s)\right] \mathrm{d} \nu_{s}
$$

From the definition (40) of $\gamma_{c}^{12}(t, s)$, it is easy to check that $\bar{v}$ satisfies the equation

$$
\bar{v}_{t}=\delta_{c} \int_{0}^{t} \bar{v}_{s} \mathrm{~d} s+\int_{0}^{t}\left[\gamma_{c}^{12}(s, s)-\Gamma_{f}^{12}(t, s)\right] \mathrm{d} \nu_{s} .
$$

Now, starting from (43) and paralleling the proof of Lemma 2.5 in [12], one can check that

$$
\int_{0}^{T}\left[q \pi_{t}^{2}(\bar{X})+r \bar{u}_{t}^{2}\right] \mathrm{d} t=q \int_{0}^{T}\left[\bar{z}_{t}^{2}+\frac{\delta_{c}+a}{\delta_{c}-a} \bar{w}_{t}^{2}\right] \mathrm{d} t+\frac{q}{\delta_{c}+a}\left(\pi_{T}(\bar{X})-\bar{z}_{T}\right)^{2},
$$

where $\bar{z}$ and $\bar{w}$ are the processes defined by (112) and (118) respectively and the difference $\pi_{T}(\bar{X})-\bar{z}_{T}$ can be represented as

$$
\pi_{T}(\bar{X})-\bar{z}_{T}=\frac{b \bar{u}_{t}+\left(\delta_{c}+a\right) \bar{w}_{t}}{\delta_{c}-a} .
$$

Moreover, since using $p_{t}=\bar{\rho}\left[\pi_{t}(\bar{X})+\bar{v}_{t}\right]$ equation (50) for $p$ can be rewritten as

$$
p_{t}=-\delta_{c} \int_{0}^{t} p_{s} \mathrm{~d} s+q \int_{0}^{t} \bar{v}_{s} \mathrm{~d} s+\int_{0}^{t} \gamma_{c}^{12}(s, s) \mathrm{d} \nu_{s},
$$

from equation (118) for $\bar{w}$, one sees that

$$
p_{t}=\bar{\rho} \bar{w}_{t}+q \int_{0}^{t} \mathrm{e}^{-\delta_{c}(t-s)} \bar{v}_{s} \mathrm{~d} s
$$


Inserting (124) into $\bar{u}_{t}=-\frac{b}{r} p_{t}$, from (123), it comes that

$$
\pi_{T}(\bar{X})-\bar{z}_{T}=-\left(\delta_{c}+a\right) \int_{0}^{t} \mathrm{e}^{-\delta_{c}(t-s)} \bar{v}_{s} \mathrm{~d} s
$$

Now, recall (see Rem. 3.2) that $\bar{v}$ can be represented as

$$
\bar{v}_{t}=\mathbb{E}\left(\int_{t}^{+\infty} \mathrm{e}^{-\delta_{c}(\tau-t)} \mathrm{d} V_{\tau}^{H} / \mathcal{Y}_{t}^{\bar{u}}\right) .
$$

Therefore, it follows from Propositions 4.2 and 4.3. in [12] that $\lim _{t \rightarrow+\infty} \frac{\bar{v}_{t}}{\sqrt{t}}=0$ a.s. and hence, from (125), we obtain immediately that

$$
\lim _{T \rightarrow+\infty} \frac{\pi_{T}(\bar{X})-\bar{z}_{T}}{\sqrt{T}}=0 \quad \text { a.s. }
$$

Then, inserting into (122) the limits given in (126) and assertions (iii) of Lemmas 5.3 and 5.4, it is easy to check that (53) is valid.

Now, observing that $\bar{X}-\pi(\bar{X})=\Delta$, assertion (ii) in Lemma 5.2 says exactly that (54) holds and it remains only to show that (55) is valid. But (53) tells in particular that $\lim \sup _{T \rightarrow+\infty} \frac{1}{T} \int_{0}^{T} \pi_{t}^{2}(\bar{X}) \mathrm{d} t<+\infty$ a.s. Hence, since of course the process $\pi(\bar{X})$ is $\left(\mathcal{Y}_{t}^{\bar{u}}\right)$-adapted, to conclude we can just apply assertion (iii) of Lemma 5.2 with $v \equiv \pi(\bar{X})$.

Proof of properties (56)-(58). Here we prove the key properties used to show that $\bar{u}$ minimizes $J_{\infty}$. We need only to work with an admissible control $u$ such that $J_{\infty}(u)<+\infty$ a.s. and so, in particular, $\lim \sup _{T \rightarrow+\infty} \frac{1}{T} \int_{0}^{T} X_{t}^{2} \mathrm{~d} t<$ $+\infty$ a.s. Since $\pi_{t}^{2}(X) \leq 2 X_{t}^{2}+2 \Delta_{t}^{2}$ and (54) holds, for such a control we have also $\lim \sup _{T \rightarrow+\infty} \frac{1}{T} \int_{0}^{T} \pi_{t}^{2}(X) \mathrm{d} t<$ $+\infty$ a.s. Hence, applying assertion (iii) in Lemma 5.2 with $v \equiv \pi(X)$, we get that

$$
\lim _{T \rightarrow+\infty} \frac{1}{T} \int_{0}^{T} \pi_{t}(X) \Delta_{t} \mathrm{~d} t=0 \quad \text { a.s. }
$$

which, due to (55), gives that (56) is also valid.

Concerning (57), we write

$$
\frac{1}{T} p_{T}\left[\pi_{T}(\bar{X})-\pi_{T}(X)\right]=\frac{p_{T}}{\sqrt{T}} \frac{\pi_{T}(\bar{X})-\pi_{T}(X)}{\sqrt{T}} .
$$

Paralleling the proof of Lemma 2.6 in [12], one can show that

$$
\limsup _{T \rightarrow+\infty}\left|\frac{\pi_{T}(\bar{X})-\pi_{T}(X)}{\sqrt{T}}\right|<+\infty \quad \text { a.s. }
$$

Moreover, since assertion $(i i)$ in Lemma 5.4 says that $\lim _{t \rightarrow+\infty} \frac{\bar{w}_{t}}{\sqrt{t}}=0$ a.s. and we know that $\lim _{t \rightarrow+\infty} \frac{\bar{v}_{t}}{\sqrt{t}}=$ 0 a.s., due to (124), we have

$$
\lim _{T \rightarrow+\infty} \frac{p_{T}}{\sqrt{T}}=0 \quad \text { a.s. }
$$

and hence (57) holds.

Now, it remains only to show that (58) is valid. We rewrite it as

$$
\lim _{T \rightarrow+\infty} \frac{N_{T}}{\langle N\rangle_{T}} \frac{\langle N\rangle_{T}}{T}=0 \quad \text { a.s. }
$$


where $\left(N_{t}, t \geq 0\right)$ is the martingale defined by

$$
N_{t}=\int_{0}^{t}\left[\pi_{s}(X)-\pi_{s}(\bar{X})\right] \gamma_{c}^{12}(s, s) \mathrm{d} \nu_{s}
$$

with the quadratic variation process $\left(\langle N\rangle_{t}, t \geq 0\right)$ given by

$$
\langle N\rangle_{t}=\int_{0}^{t}\left[\pi_{s}(X)-\pi_{s}(\bar{X})\right]^{2}\left[\gamma_{c}^{12}(s, s)\right]^{2} \mathrm{~d} w_{s}^{H} .
$$

Due to (127) and assertion (i) in Lemma 5.4, we have

$$
\limsup _{T \rightarrow+\infty} \frac{\langle N\rangle_{T}}{T}<+\infty \quad \text { a.s. on }\left\{\langle N\rangle_{T} \rightarrow+\infty\right\},
$$

and so (58) follows immediately from Lemma 2.6 .3 in [14].

\section{REFERENCES}

[1] F. Biagini, Y. Hu, B. Øksendal, and A. Sulem, A stochastic maximum principle for processes driven by fractional Brownian motion. Stoch. Processes Appl. 100 (2002) 233-253.

[2] H. Cramer and M.R. Leadbetter, Stationary and related stochastic processes. John Wiley \& Sons, Inc. (1967).

[3] M.H.A. Davis, Linear Estimation and Stochastic Control. Chapman and Hall (1977).

[4] L. Decreusefond and A.S. Üstünel, Stochastic analysis of the fractional Brownian motion. Potential Analysis 10 (1999) $177-214$.

[5] T.E. Duncan, Y. Hu and B. Pasik-Duncan, Stochastic calculus for fractional Brownian motion I. Theory. SIAM J. Control Optim. 38 (2000) 582-612.

[6] G. Gripenberg and I. Norros, On the prediction of fractional Brownian motion. J. Appl. Prob. 33 (1996) 400-410.

[7] M.L. Kleptsyna and A. Le Breton, Statistical analysis of the fractional Ornstein-Uhlenbeck type process. Stat. Inf. Stoch. Processes 5 (2002) 229-248.

[8] M.L. Kleptsyna and A. Le Breton, Extension of the Kalman-Bucy filter to elementary linear systems with fractional Brownian noises. Stat. Inf. Stoch. Processes 5 (2002) 249-271.

[9] M.L. Kleptsyna, A. Le Breton and M.C. Roubaud, General approach to filtering with fractional Brownian noises - Application to linear systems. Stoch. Stoch. Reports 71 (2000) 119-140.

[10] M.L. Kleptsyna, A. Le Breton and M. Viot, About the linear-quadratic regulator problem under a fractional Brownian perturbation. ESAIM: PS $\mathbf{7}$ (2003) 161-170.

[11] M.L. Kleptsyna, A. Le Breton and M. Viot, Asymptotically optimal filtering in linear systems with fractional Brownian noises. Stat. Oper. Res. Trans. 28 (2004) 177-190.

[12] M.L. Kleptsyna, A. Le Breton and M. Viot, On the infinite time horizon linear-quadratic regulator problem under a fractional Brownian perturbation. ESAIM: PS 9 (2005) 185-205.

[13] R.S. Liptser and A.N. Shiryaev, Statistics of Random Processes. Springer-Verlag (1978).

[14] R.S. Liptser and A.N. Shiryaev, Theory of Martingales. Kluwer Academic Publ., Dordrecht (1989).

[15] I. Norros, E. Valkeila and J. Virtamo, An elementary approach to a Girsanov formula and other analytical results on fractional Brownian motions. Bernoulli 5 (1999) 571-587.

[16] C.J. Nuzman and H.V. Poor, Linear estimation of self-similar processes via Lamperti's transformation. J. Appl. Prob. 37 (2000) 429-452.

[17] W.M. Wonham, On the separation principle of stochastic control. SIAM J. Control 6 (1968) 312-326. 